\title{
Application of Carbonene Materials for Artificial Muscles
}

\author{
Yeye Wen ${ }^{1,2}$, Ming Ren ${ }^{3,4}$, Jiangtao Di ${ }^{3,4}$, Jin Zhang ${ }^{1,2, *}$ \\ ${ }^{1}$ Center of Nano Chemistry, Beijing National Laboratory for Molecular Sciences, College of Chemistry and Molecular Engineering, \\ Peking University, Beijing 100871, China. \\ ${ }^{2}$ Beijing Graphene Institute (BGI), Beijing 100095, China. \\ ${ }^{3}$ Suzhou Institute of Nano-Tech and Nano-Bionics, Chinese Academy of Sciences, Suzhou 215123, Jiangsu Province, China. \\ ${ }^{4}$ School of Nano-Technology and Nano-Bionics, University of Science and Technology of China, Hefei 230026, China.
}

\begin{abstract}
The development of new types of artificial muscles is of utmost importance as traditional actuators based on mechanical drive systems no longer meet the stringent requirements of flexibility, high efficiency, and multistimuli responses in advanced functional fields, such as soft and biomimetic robots, sensors, artificial intelligent control, and artificial intelligence. Carbonene materials refer to carbon materials composed of all carbon atoms with $s p^{2}$ hybridization, mainly including carbon nanotubes and graphene. Owing to their exceptional properties such as light weight, excellent mechanical performance, high conductivity, flexibility, and large specific surface area, carbonene materials demonstrate significant application potential in artificial muscles, thereby promoting the rapid development of corresponding fields. Herein, the recent progress of the application of carbonene materials in artificial muscles is summarized to provide a comprehensive understanding of the preparation, properties, and applications of artificial muscles composed of carbonene materials. First, carbonene artificial muscles integrating response, actuation, and structure are introduced. As carbonene materials are unique building blocks that can be readily assembled into macroscopic materials with various structures, fibrous and membranous artificial muscles based on carbonene materials are discussed in detail. Carbonene fiber actuators demonstrate diverse actuation performances when fabricated with different structures. Bending actuation typically occurs when carbonene artificial muscles with asymmetric structures are subjected to external stimulation. The untwisting of carbonene artificial muscle fibers with twisted structures causes torsional and tensile actuation, which can be attributed to the volume expansion induced by external stimuli. Furthermore, coiled structures achieved by twisting a fiber until it is fully coiled can enhance the actuation stroke. Thus, the actuation of artificial muscle fibers made of carbonene materials can be classified into bending, rotation, and contraction actuations. Second, carbonene materials have long been considered as a functional component in composite materials for specific applications owing to their excellent physical and chemical properties. Therefore, the application of carbonene materials as an additional component to other artificial muscle materials (such as smart hydrogels, dielectric elastomers, and conducting polymers) is reviewed. By employing carbonene materials, artificial muscle materials exhibit improved electrical and mechanical properties, thereby leading to superior actuation performances. In addition, integrating carbonene materials into artificial muscles can endow the muscles with programmable actuation and sensing functions. Finally, the challenges faced in the application of artificial muscles based on carbonene materials and the future application of carbonene artificial muscles with multi-functional actuation performance are briefly discussed.
\end{abstract}

Key Words: Carbonene materials; Artificial muscles; Application; Structure; Function

Received: July 2, 2021; Revised: July 28, 2021; Accepted: July 28, 2021; Published online: August 5, 2021.

${ }^{\star}$ Corresponding author. Email: jinzhang@pku.edu.cn.

The project was supported by the Beijing National Laboratory for Molecular Sciences (BNLMS-CXTD-202001), the Ministry of Science and Technology of China (2016YFA0200100, 2018YFA0703502), and the National Natural Science Foundation of China (52021006, 51720105003, 21790052, 21974004). 北京分子科学国家研究中心(BNLMS-CXTD-202001), 中国科学技术部(2016YFA0200100, 2018YFA0703502), 国家自然科学基金(52021006, $51720105003,21790052,21974004)$ 资助项目

(C) Editorial office of Acta Physico-Chimica Sinica 


\title{
烯碳材料在人工肌肉领域的应用进展
}

\author{
温烨烨 1,2, 任明 3,4 , 邸江涛 3,4 , 张锦 1,2, \\ 1 北京大学化学与分子工程学院, 北京分子科学国家研究中心, 北京大学纳米化学研究中心, 北京 100871 \\ 2 北京石墨烯研究院, 北京 100095 \\ 3 中国科学院苏州纳米技术与纳米仿生研究所, 江苏苏州 215123 \\ 4 中国科学技术大学纳米技术与纳米仿生学院, 合肥 230026
}

\begin{abstract}
摘要: 随着仿生机器人、智能控制及人工智能等领域的发展, 传统的机械驱动方式已无法满足相关领域对致动系统提出 的柔性、高效及多源刺激响应性等要求, 因此需发展新型的人工肌肉材料。以碳纳米管和石墨烯为代表的烯碳材料具有 轻质、高强、高电导率和柔性等特征, 在人工肌肉领域展现出了巨大的应用潜力。以烯碳材料为基元构筑宏观组装体材 料, 或以烯碳材料为添加相制备纳米复合材料, 可在微观和宏观架起桥梁, 实现烯碳材料在人工肌肉领域的应用。本文 基于上述两种应用形式, 综述了烯碳材料在人工肌肉领域的应用进展。首先从一维纤维和二维薄膜的烯碳人工肌肉宏观 表现形态出发, 介绍了既作为结构材料, 又提供了响应、驱动功能的烯碳材料在人工肌肉中的应用。接着从机电性能、 可编程的响应形变以及传感功能三个方向, 介绍了烯碳材料作为增强赋能相在人工肌肉材料中的功能性应用。最后阐述 了基于烯碳材料人工肌肉的机遇与挑战。
\end{abstract}

关键词: 烯碳材料; 人工肌肉; 应用; 结构; 功能

中图分类号: 0647

\section{1 引言}

人工肌肉是一类受外界刺激(光、电、热等)发 生可逆响应形变的智能材料和系统, 又称驱动器 ${ }^{1}$ 。 依赖机械传动装置的传统驱动器, 例如液压/气动 驱动器, 是在工业中广泛应用的人工肌肉系统。其 具有负载大、可输出高力矩、动作迅速反应快的优 点, 但结构复杂且难以小型化。另一种具有广泛商 业应用的传统人工肌肉是基于压电陶瓷的精确控 制机构一一压电驱动器, 所产生的精确、极小的形 变量使其可用在对仪器及控制精密度有高要求的 微电子技术和生物工程等领域 ${ }^{2}$, 但同时也严格限 制了使用场景。随着包含物联网、人机交互界面、 仿生机器人及智能穿戴等领域的发展, 传统刚性 驱动器已无法满足先进应用领域对人工肌肉柔 性、轻质、具有环境适应性以及高的能量转换效率 等新要求 3,4 。因此需开发集响应、驱动、结构等功

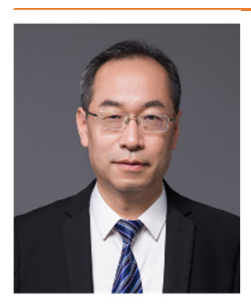

张锦, 中国科学院院士、北京大学教授、 博士生导师/国家杰出青年基金获得 者、教育部长江学者特聘教授、英国皇 家化学学会会士、中组部 “万人计划” 创新领军人才入选者、科技部重点研发 计划项目负责人。1997年获兰州大学理 学博士学位, 1998年至2000年在英国利兹大学从事博士后 研究, 2000年5月到北京大学工作。主要从事纳米碳材料 的控制制备与拉曼光谱学研究。
能为一体的人工肌肉材料。

得益于材料科学的蓬勃发展, 科学家们探索、 制备了具有与生物肌肉相似运动功能的仿生人工 肌肉材料。生物肌肉由一束肌肉纤维组成, 虽然肌 肉纤维在刺激下可产生 $50 \%$ 的收缩, 但在大多数动 物中, 由于受到所连接关节的限制, 该收缩形变通 常小于 $20 \%$, 且一块肌肉能产生的输出功率主要在 0.1- $0.5 \mathrm{~W} \cdot \mathrm{g}^{-1}$ 之间 ${ }^{1}$ 。人工肌肉由生物肌肉所启发, 却具备远超于生物肌肉的驱动性能和更多元的驱 动形式。新材料及其制造技术为能有效实现电能、 化学能、热能或光能等转化成机械能的人工肌肉 的制备提供了多种选择, 并发展出基于纳米复合 材料 5 、形状记忆合金及聚合物 6,7 、介电弹性体 8 、 离子聚合物/金属复合材料 ${ }^{9}$ 、导电聚合物 ${ }^{10,11}$ 生物 质纤维 ${ }^{12-14}$ 等智能材料的人工肌肉 1 。其中, 伴随着 纳米材料的制备和表征技术的发展, 特别是烯碳 材料在制备、结构和性能上的巨大突破，由烯碳材 料构筑而成的人工肌肉的相关研究推动了该领域 的重大发展 $5,15-17$ 。

烯碳材料是指由 $s p^{2}$ 杂化碳原子组成的全碳材 料, 主要包括碳纳米管(CNT)和石墨烯。由于碳碳 双键的键连方式, 理想的CNT与石墨烯具有超高 的拉伸强度 $(>100 \mathrm{GPa})$ 、杨氏模量 $(\sim 1 \mathrm{TPa})$ 、高热 导率 $\left(>3000 \mathrm{~W} \cdot \mathrm{m} \cdot \mathrm{K}^{-1}\right)$ 、高电导率 $\left(10^{8} \mathrm{~S} \cdot \mathrm{m}^{-1}\right)$ 和巨 大的比表面积等特性 ${ }^{18-20}$ 。将烯碳材料应用于人工 肌肉领域, 需在微观和宏观尺度架起桥梁。一方面 
以烯碳材料为基本结构单元, 利用多种组装方法 构筑烯碳材料宏观组装体, 通过组装结构的设计 可将烯碳人工肌肉微观结构的体积形变转化为弯 曲、旋转或伸缩驱动, 从而实现集响应、驱动和结 构一体化人工肌肉的制备 $5,21,22$ 。另一方面可采用 烯碳材料为增强赋能的添加相, 通过复合策略, 优 化形状记忆聚合物、导电高分子、智能水凝胶等人 工肌肉材料的驱动性能, 制备基于纳米复合材料 的多功能人工肌肉 ${ }^{1}$ 。由于诸多优异的理化性质, 烯碳材料在人工肌肉中具有独特的应用。例如以 具有捻曲结构的CNT纱线为主体材料制备纤维状 人工肌肉, 当复合热变形的客体材料以实现纤维 的热致驱动时, 得益于 $\mathrm{CNT}$ 的电热、光热转化能 力, 人工肌肉纤维还可在外加电压和光照下发生 形变, 具有多源刺激响应性 ${ }^{23}$ 。在产生驱动形变时, CNT纱线既作为人工肌肉的结构材料, 又起到了 能量转化的作用, 因此集响应、驱动、结构功能为 一体。随着应用场景的不断革新, 人工肌肉也向轻 量、柔性以及结构-功能-智能一体化发展。由于自 身优异的理化特性, 烯碳材料在人工肌肉领域展 现出巨大的应用潜力。

本文从烯碳材料的结构及性能出发, 通过介 绍烯碳材料在人工肌肉中的两大应用方向, 综述 了烯碳材料在人工肌肉领域的应用进展。首先基 于烯碳人工肌肉的宏观表现形态(一维纤维和二 维薄膜), 介绍了烯碳材料组装体在人工肌肉中的 应用。接着介绍了烯碳材料作为增强赋能的添加 相, 在纳米复合材料人工肌肉中的应用。最后对人 工肌肉的未来发展进行了展望。

\section{2 烯碳材料人工肌肉}

目前科学家通过各异的途径与手段, 将烯碳 材料可控组装成形式多样的宏观材料, 实现其性 能从微观到宏观的传递。2002年, 清华大学范守善 教授团队 24 通过CNT阵列纺丝法, 即从高度取向的 可纺碳纳米管坚直阵列中, 连续拉出 $\mathrm{CNT}$, 首次制 备了 $\mathrm{CNT}$ 纤维。随后Zhang 等 ${ }^{25}$ 报道了一种基于 CNT可纺阵列的拉伸加捻纺丝法, 制备碳纳米管 纺线。目前, 阵列纺丝法、化学气相沉积直接纺丝 法以及湿法纺丝法是 CNT纤维的主要制备方法 ${ }^{26}$ 。 2011 , 浙江大学高超教授团队 27 发现了氧化石墨烯 (GO)的溶致液晶现象, 通过湿法纺丝和溶液还原 的方法首次制备了石墨烯纤维。随之发展出基于 $\mathrm{GO}$ 溶液的干法纺丝、限域水热法以及薄膜加捻法 等石墨烯纤维制备方法 ${ }^{28}$ 。由于CNT以及石墨烯材 料的柔性以及高强度的特征, 可通过纤维制备过
程直接加捻或者对成型纤维后加捻的方法, 使 CNT或石墨烯纤维具有一定捻曲结构 17,29 。除了宏 观的一维纤维材料以外, CNT与石墨烯还可通过 多种制备方法组装成二维薄膜材料 ${ }^{30,31}$ 。以烯碳材 料为基本构筑基元的纤维、薄膜在展现出优异力 学性能的同时, 还兼具高电导率、高热导率等优 点, 且组装体的结构及性能可调控性高, 因此在要 求材料具有结构功能一体化的领域具有极大的应 用潜力。烯碳纤维及薄膜制备技术的发展, 为基于 烯碳材料的高性能人工肌肉的设计与制备奠定了 坚实的基础。

烯碳材料组装结构和微观形貌的差异, 使其 作为人工肌肉应用时, 展现出不同的驱动形式及 驱动性能。本节将基于烯碳材料人工肌肉的宏观 表现形态, 包含一维纤维和二维薄膜, 讨论烯碳材 料在集响应、驱动和结构功能为一体的人工肌肉 中的应用。

\section{1 纤维状烯碳人工肌肉}

人工肌肉材料在外加刺激下发生的可逆体 积形变, 是其产生驱动行为的基础。2004年, Baughman教授团队 ${ }^{32}$ 采用湿法纺丝技术制备了单 壁碳纳米管/聚乙烯醇(SWNT/PVA) 纤维, 通过热 解除去PVA后, 所得纤维在电化学池中受外加电 压刺激发生体积膨胀/收缩, 产生高达 $26 \mathrm{MPa}$ 的应 力 (自然肌肉的 100 倍), 该形变主要来源于离子在 纤维结构内部的可逆注入与排出。利用DNA使 $\mathrm{SWNT}$ 解缠结可增加 CNT 纤维与离子作用的有效 面积, 并实现SWNT的交联, 所得DNA/SWNT复合 纤维受电化学驱动可产生 $0.12 \%$ 的体积膨胀与收 缩(图1a) ${ }^{33}$ 。相比于上述SWNT纤维, DNA的交联 降低了复合纤维的蠕变行为, 提高了驱动的可逆 性(图1b)。随后通过复合聚吡咯(PPy) ${ }^{34}$, 纤维素 ${ }^{35}$ 、 碳颗粒 ${ }^{36}$ 等材料, 发展出多种具有膨胀/收缩形变 的CNT复合纤维人工肌肉。实际上, 受结构限制, 这一类 CNT纤维材料的驱动行为仅来源于简单纤 维结构的体积膨胀与收缩, 因此产生的形变量小 (图1b), 驱动效果差, 难以满足复杂应用场景的需 求。

烯碳材料的组装和纤维制备技术的发展, 催 生了新型纤维状烯碳人工肌肉。得益于特殊的组 装结构, 新型纤维状烯碳人工肌肉可将材料的体 积膨胀与收缩扩展为更丰富的驱动形式(弯曲、旋 转和伸缩驱动) 并获得优异的驱动效果。例如通过 构筑纤维的不对称结构, 可制备具有大弯曲形变 的烯碳纤维。在烯碳纤维中引入捻曲结构, 可将外 界刺激引起的体积形变转换为纤维径向的转动和 
(a)

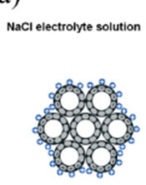

(b)

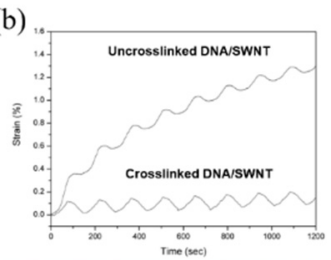

(c)

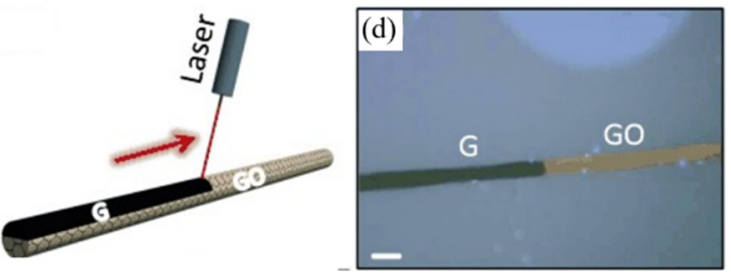

(e)

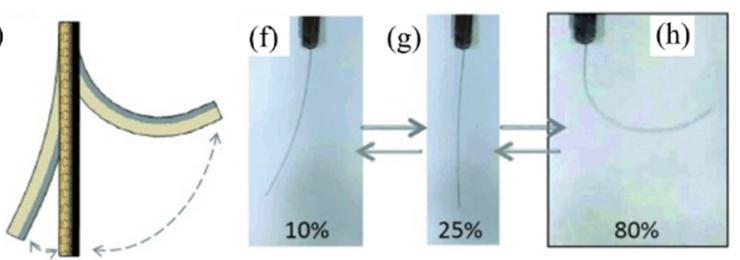

图 1 膨胀收缩和弯曲驱动的烯碳纤维人工肌肉

Fig. 1 Carbonene artificial muscle fibers with expansion/shrinkage and bending actuation.

(a) Charge injection in a nanotube bundle (left) and a DNA/SWNT hybrid system with unbundled SWNT (right). (b) Plot of strain versus time of uncrosslinked and crosslinked DNA/SWNT hybrid fiber during cycling voltammetry. Adapted with permission from Ref. 33. Copyright 2008, Wiley-

VCH. (c) Schematic illustration of positioned laser reduction on one side of a GO fiber. (d) Photomicrograph of the top surface of the asprepared asymmetric $\mathrm{G} / \mathrm{GO}$ fiber. (e) Representation of the possible bending of a G/GO fiber exposed to different relative humidities. (f-h) Photographs of a $\mathrm{G} / \mathrm{GO}$ fiber ( $2 \mathrm{~cm}$ in length) under different relative humidities.

Adapted with permission from Ref. 37. Copyright 2013, Wiley-VCH.

轴向的收缩, 从而发生旋转和伸缩驱动。烯碳纤维 人工肌肉的运动形式取决于组装结构, 因此本节 将按照人工肌肉运动形式的分类(弯曲、旋转和伸 缩驱动), 介绍具有不同组装结构的烯碳材料在纤 维状人工肌肉中的应用。

\subsection{1 弯曲驱动的烯碳纤维人工肌肉}

Cheng等 ${ }^{37}$ 用激光加工 $G O$ 纤维, 使得部分区域 还原, 构筑具有不对称结构的石墨烯/氧化石墨烯 (G/GO)纤维(图1c, d)。石墨烯和氧化石墨烯片层 上含氧官能团数量的差异, 造成两者具有截然不 同的亲疏水性能。因此当暴露在湿度环境下, 亲水 的 $\mathrm{GO}$ 组分吸附水分子发生体积膨胀, 而 $\mathrm{G}$ 组分不 发生明显形变, 由于纤维两侧表面形变量不一致, 产生内部应力, 纤维发生弯曲, 即实现弯曲驱动 (图1e-h)。将纤维的一端固定, 当环境相对湿度从 $25 \%$ 提升到 $80 \%$ 时, 纤维的弯曲角度可由 $0 \circ$ 迅速增
加至 $140^{\circ}$ 。Wang 等 ${ }^{38}$ 在石墨烯纤维一侧电镀一层 PPy, 制备了具有不对称结构的石墨烯/PPy复合纤 维。导电高分子PPy在不同电压下进行掺杂与去掺 杂时, 可通过离子的迁移产生体积形变, 而石墨烯 在电解液中由于电化学双电层充放电过程, 也会 产生体积形变。因此石墨烯/PPy复合膜可受外加 电压刺激发生弯曲驱动。石墨烯在复合纤维中既 作为导电组分, 又是驱动的主体材料。

不对称结构的设计是人工肌肉产生弯曲驱动 的关键。对于纤维状人工肌肉而言, 其弯曲驱动的 研究并不多, 学者更关注具有更高能量转换效率、 功率密度的旋转和伸缩致动。更多的关于弯曲致 动的结构设计和应用将在下节二维薄膜状烯碳人 工肌肉部分进行介绍。

\subsection{2 旋转驱动的烯碳纤维人工肌肉}

Baughman教授团队 ${ }^{17}$ 在人工肌肉领域取得许 多开创性的成果。2011年, 他们首先报道了可用作 旋转驱动器的 CNT纱线。随着捻度增加, 具有捻曲 结构的CNT纱线内部产生扭矩, 而纱线可通过体 积变化实现内部扭矩的平衡。此时, 解捻的过程使 纤维结构发生变化, 产生旋转驱动(图 $2 a, b)$ 。值得 注意的是, 仅有纤维状的人工肌肉才可发生旋转 驱动。

将扭曲的CNT纱线作为工作电极浸入电解液 中, 电解质离子和溶剂化物质在外加电压作用下, 从电解液迁入 $\mathrm{CNT}$ 纱线内部引起纱线体积膨胀和 结构解捻, 驱使 CNT纱线发生转动 ${ }^{17}$ 。若 CNT纱线 仅是一端固定, 另一端连着可自由转动的旋浆(图 $2 \mathrm{c})$, 该人工肌肉需经过多次循环后才可获得可逆 的旋转驱动, 而该过程降低了旋转驱动的驱动量。 为了提高转动的可逆性, 他们开发了一种两端固 定的 CNT纱线人工肌肉构型, 即将 CNT纱线下半 部分浸入电解液中, 下端完全固定, 上端连接一个 力/距离传感器, 可上下移动并保持固定应力(图 2d)。当浸入电解液的CNT纱线发生解捻时, 下部 分失去的捻度作为上捻转移到上部分。撤去外加 电压后, 上部分 CNT纱线可作为复位弹簧, 将捻度 传回下部分, 从而实现完全的可逆旋转。若将可自 由旋转的旋浆置于两部分中间, 则旋浆可在旋 转驱动和回复的过程中发生相反方向的旋转。该 $\mathrm{CNT}$ 人工肌肉能够在 $1.2 \mathrm{~s}$ 内加速旋浆转动(驱动电 压 $5 \mathrm{~V})$, 转速高达 $590 \mathrm{r} \cdot \mathrm{min}^{-1}$, 产生与大型商用电 机可比的单位质量的力矩和机械功率, 可用作微 流体搅拌器件(图2e)。但该人工肌肉在 $88 \mathrm{MPa}$ 载荷 下仅产生 $1 \%$ 的收缩驱动。由此可见, 这类加捻结 构可产生良好的转动驱动效果, 但收缩驱动量 
(a)

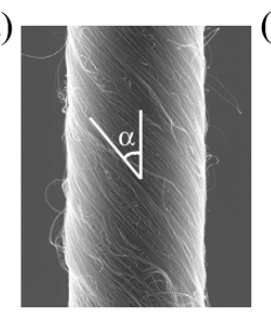

(f)

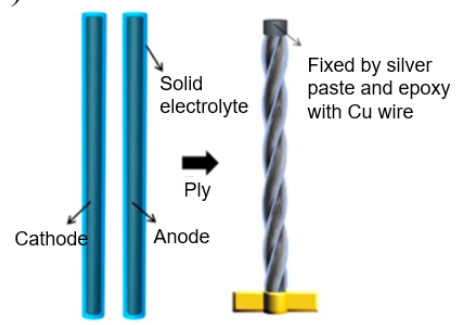

(i)

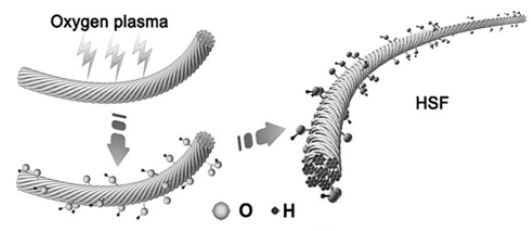

(c)
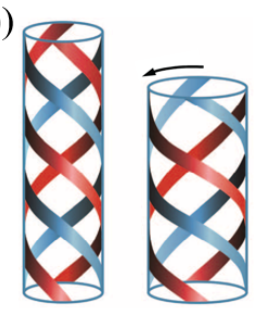

(g)

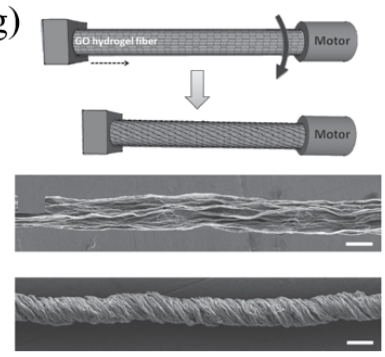

(j)

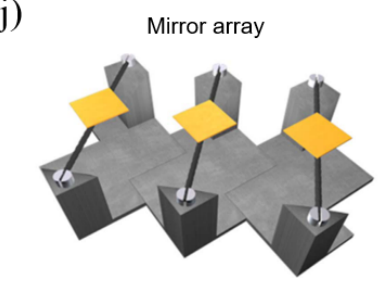

(d)

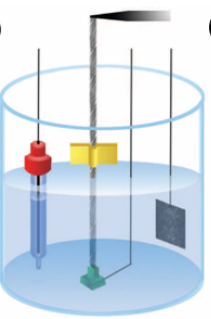

(e)

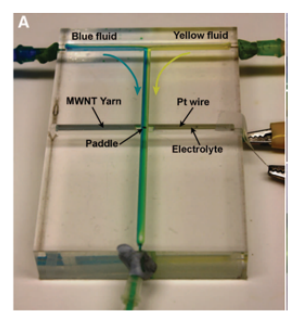

(h)
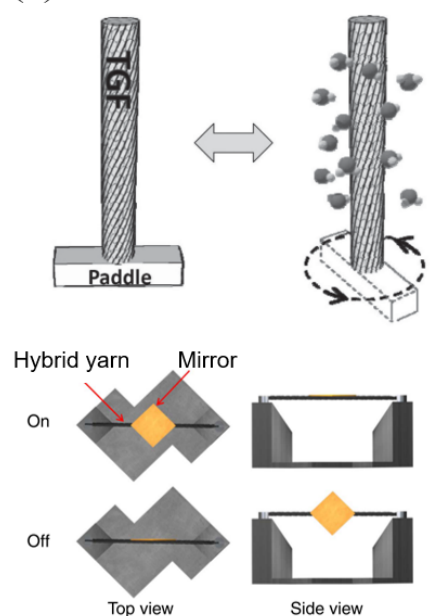

图 2 旋转驱动的烯碳纤维人工肌肉

Fig. 2 Carbonene artificial muscle fibers with torsional actuation.

(a) Twisted structure of CNT yarns. (b) Schematic illustration of the effect of volume expansion on the twist and length of the yarn, the arrow indicates the untwisting direction of yarn. (c) A one end-tethered yarn configuration with a paddle located at the yarn end. (d) A two-end-tethered configuration with a force/distance transducer at the upper end that maintains constant tensile force on the yarn and measures the axial length change. (e) Photograph of prototype mixer that can be downscaled for a microfluidic circuit. Adapted with permission from Ref. 17. Copyright 2011, AAAS.

(f) Plying anode and cathode yarns which infiltrated and coated with PVDF-co-HFP based TEABF 4 solid gel electrolyte to make all-solid-state CNT torsional artificial muscle. Adapted with permission from Ref. 39. Copyright 2014, American Chemical Society. (g) Scheme of the torsional graphene-fiber motor (TGF) fabrication (top), SEM images of directly dried GO fiber (middle) and TGF with an applied 5000 turns per meter (down). (h) Schematic rotation of a TGF at the low (left) and high (right) humidity. Adapted with permission from Ref. 29. Copyright 2014, Wiley-VCH. (i) Plasma modification of CNT fiber with hierarchically helical channels, which responses to water and moisture. Adapted with permission from Ref. 40. Copyright 2015, Wiley-VCH.

(j) Schematic illustration of the demonstrated use of torsional yarn muscle to precisely rotate mirrors without producing significant torsional oscillations. Adapted with permission from Ref. 41. Copyright 2014, Nature Publishing Group.

小。因此加捻结构一般用作旋转驱动器的结构设 计中。

上述电化学驱动的人工肌肉虽然仅需较低电 压就可产生驱动效果, 但使用时需依赖电解液。电 解液较大的体积与质量, 降低了整个驱动系统的 体积和能量密度。Lee等 39 将涂覆有凝胶电解质的 两条低捻度CNT纱线摚合在一起, 实现阳极和阴 极人工肌肉驱动行程的匹配, 得到在环境中工作 的旋转驱动全固态人工肌肉(图2f)。该人工肌肉在 $5 \mathrm{~V}$ 的外加方波电压下, 转速可达 $2330 \mathrm{r} \cdot \mathrm{min}^{-1}$ 。

具有加捻结构烯碳纤维的转动驱动来源于纤 维内部结构间隙扩大而产生的解捻, 因此在设计 具有加捻结构的纤维状人工肌肉时, 可采用加捻
的烯碳纤维为主体结构, 利用其他能够在其结构 间隙中可逆注入与脱除的分子 (例如水) ${ }^{29,40}$ 作为 外界刺激, 实现可逆转动驱动, 另一个途径则是以 能够在外界刺激下发生可逆形变的材料为客体材 料, 与加捻烯碳纤维复合, 从而制备得到具有不同 刺激响应的转动驱动人工肌肉 $23,41-46$ 。

Cheng等 ${ }^{29}$ 对 GO气凝胶进行加捻, 制备湿度驱 动的转动人工肌肉(图 $2 g, h$ )。由于 $G O$ 表面具有丰 富的羧基、羟基等含氧官能团, 使其可快速吸附环 境湿度中的水分子, 产生体积膨胀, 发生转动驱 动, 该湿度驱动的人工肌肉在 $3.5 \mathrm{~s}$ 内转速可达 $5190 \mathrm{r} \cdot \mathrm{min}^{-1}$ 。利用氧等离子体对加捻的 CNT纱线 进行表面亲水化处理, 同样可以获得对水/蒸汽具 
有响应性的转动 $\mathrm{CNT}$ 人工肌肉(图 $2 \mathrm{i})^{40}$ 。

Baughman教授团队 ${ }^{23}$ 以具有高热膨胀系数的 石蜡为客体材料, 制备了CNT复合纱线。该人工肌 肉可在 $15 \mathrm{~Hz}, 40 \mathrm{~V} \cdot \mathrm{cm}^{-1}$ 的方波电压刺激下循环驱 动 200 万次, 且平均转速达 $11500 \mathrm{r} \cdot \mathrm{min}^{-1}$ 。随后他 们将石蜡和聚苯乙烯-聚 (乙烯-丁烯)-聚苯乙烯 (SEBS)共聚物复合, 优化客体材料组分, 制备了具 有精确和快速定位的旋转人工肌肉, 转速最高达 $9800 \mathrm{r} \cdot \mathrm{min}^{-1}$, 并展示了该人工肌肉在旋转镜阵列(可 快速在水平/垂直镜面间切换)中的应用(图 $2 \mathrm{j})^{41}$ 。 此外, 客体材料还可扩展为聚丁二炔、聚乙二醇 (电热驱动)、钯(氢气吸脱附驱动) 23 、聚丙烯酸钠 (近红外光驱动) 42 、海藻酸钠 (光驱动) 43 、含葡萄 糖氧化酶的热响应水凝胶聚 $N$-异丙基丙烯酰胺 (PNIPAm, 由于酶的放热催化效应, 复合人工肌肉 可由葡萄糖引起体积形变) ${ }^{44}$ 等材料, 丰富了具有 转动驱动人工肌肉纤维的应用场景, 例如用作检 测湿度、气体、生物分子的传感器。

在制备具有主客体结构的烯碳纤维人工肌肉 时, 除了采用直接浸润法实现客体材料与烯碳纤 维的复合外, 还可匹配烯碳纤维的干法和湿法纺 丝过程制备复合纱线 45,46 。针对不适用于干法、湿 法过程的客体材料, Lima等 ${ }^{47}$ 开发出主客体双纺法 制备复合CNT纱线, 即利用从CNT坚直阵列中抽 出的 CNT气凝胶薄膜具有高机械强度及大比表面 积的特征, 将客体材料附着在薄膜表面, 结合后续 的加捻纺丝过程, 制备复合CNT纱线(图3a-d)。

\section{1 .3 伸缩驱动的烯碳纤维人工肌肉}

Zhang等 ${ }^{25}$ 从坚直CNT阵列中抽膜、加捻并编
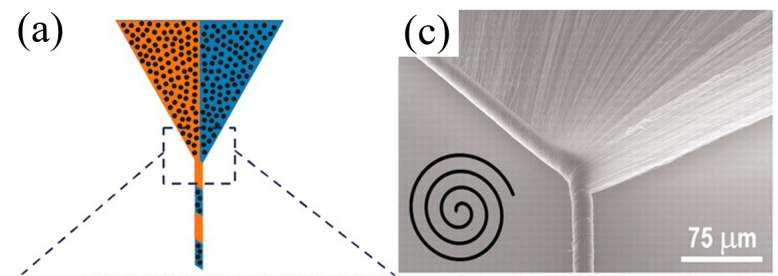

(b)
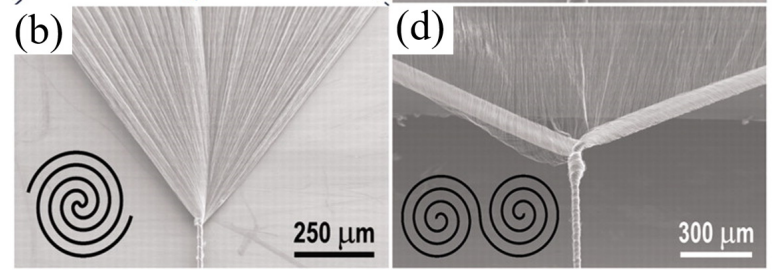

图 3 主客体双纺法制备复合 CNT 纱线

Fig. 3 Fabrication of composite carbonene yarns by biscrolling.

(a) Biscrolling of CNT sheets and functional guests (black dots). SEM micrograph of (b) Fermat scroll (c) Archimedean and (d) dual Archimedean scrolls. Adapted with permission from Ref. 47. Copyright 2011, AAAS.
织得到加捻 $\mathrm{CNT}$ 的单纱纺线、两股的 CNT螺旋纺 线、四股、针织和打结CNT纺线, 并深入研究其力 学性能。加捻及编织后的CNT纺线具有高韧性, 有 趣的是, 螺旋结构的CNT纺线还具有大的泊松比。 随着应变增大, 加捻 $\mathrm{CNT}$ 纺线的泊松比由 2.0 增加 至 2.7 , 而两股的CNT螺旋纺线则由 3.3 增大至 4.2 (图4a-c)。即螺旋结构可进一步放大纺线在截面/ 体积变化下, 引起的长度方向的形变, 为通过体积 变化实现大应变的伸缩驱动人工肌肉的制备提供 了结构基础。而过度加捻的纤维可自发地形成螺 旋结构(图4d) ${ }^{39}$ 。基于螺旋结构人工肌肉的伸缩形

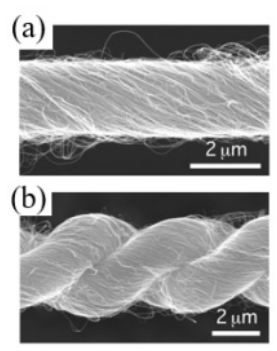

(d)

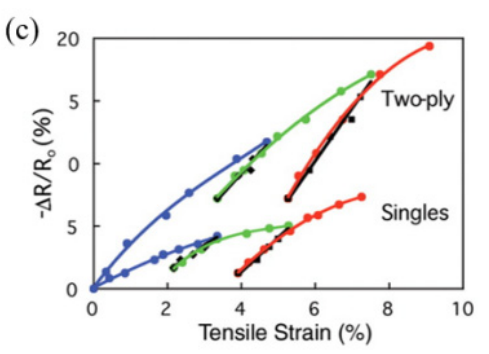

(e)
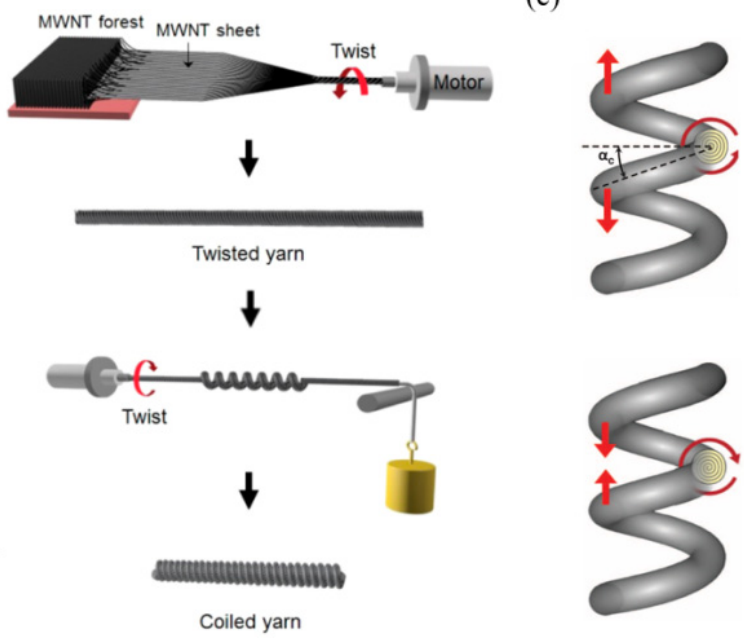

图 4 螺旋结构纱线的力学性能、制备及驱动

Fig. 4 Mechanical properties, fabrication and tensile actuation of coiled fibers.

(a) Singles and (b) two-ply of CNT yarn. (c) Percent change in diameter and length of CNT yarns with different structures as shown in (a) and (b). Symbols: blue circles, initial stretch; green circles, second stress increase; black squares, second stress decrease; red circles, stress increase until yarn rupture. Adapted with permission from Ref. 25. Copyright 2004,

AAAS. (d) Fabrication of a coiled CNT fiber from a MWNT forest.

Adapted with permission from Ref. 39. Copyright 2014, American

Chemical Society. (e) Schematic illustration of the mechanism by which

torsional fiber actuation leads to large-stroke tensile actuation for fibers with heterochiral (up) and homochiral (down) coiled structure. Adapted with permission from Ref. 48. Copyright 2014, AAAS. 
变本质也是捻曲结构的解捻, 因此为了获得伸缩 形变, 需防止纤维纱线产生旋转, 才可通过螺旋结 构将径向的转动转化为轴向的收缩。顺着纤维扭 转方向进一步过度扭曲得到的加捻螺旋线圈具有 同手性结构, 而旋向与纤维扭转方向相反的则具 有异手性结构。对于同手性结构的螺旋线圈, 当内 部产生解旋力矩时, 螺旋线圈间距收缩变小, 发生 收缩变形; 而异手性结构的螺旋线圈则发生伸长 变形(图4e) 48 。

Lee等 ${ }^{39}$ 将两根涂覆有凝胶电解质(硫酸/PVA) 的螺旋 CNT纱线绞合, 制备的全固态伸缩驱动人 工肌肉最大收缩形变为 $1.3 \%$, 可拉动自身重量 25 倍的重物(图 $5 \mathrm{a}$ )。随后Lee等 49 从电化学驱动人工肌 肉的原理出发, 即电荷注入导致纱线体积膨胀, 提 出由于大的离子半径, 有机电解质可为电化学驱 动人工肌肉提供更高的体积形变以优化驱动效 率。他们探讨了不同有机电解质对驱动效果的影 响, 发现具有螺旋结构的CNT纱线在液态的四氟 磀酸四乙基铵盐 $\left(\mathrm{TEA} \cdot \mathrm{BF}_{4}\right)$ 电解质中可获得高达 $16.5 \%$ 的收缩形变 (图 $5 \mathrm{~b}$ )。进一步引入固体电解质, 设计具有平行和编织结构的人工肌肉(图5c, d), 其 收缩形变分别达 $11.6 \%$ 和 $5 \%$ 。在电化学驱动人工 肌肉中, 有机电解质虽具有高电化学窗口和大离 子半径的优点, 但其使用也受限于自身的毒性、高 价格、低离子电导率的缺点。因此也发展出具有快 速驱动响应, 基于无机液体电解质的人工肌肉 50 。 由于反向离子的嵌入和迁出, 电化学驱动 $\mathrm{CNT}$ 人 工肌肉的体积变化不随扫描电压发生单调性变 化, 即驱动应变与扫描电压间呈现 “双极” 关系, 且驱动性能随扫描速率增加而降低。Chu等 ${ }^{51}$ 通过 聚电解质功能化策略, 改变了电化学驱动 $\mathrm{CNT}$ 人 工肌肉的零电位点, 实现了单一离子的嵌入与迁 出 (图 $5 \mathrm{e}, \mathrm{f}$ ), 并解决了驱动性能对工作电极电 容特性的依赖。制备的人工肌肉能够在高频下响 应 $(10 \mathrm{~Hz})$, 且在低电压下 $(1 \mathrm{~V})$ 可实现高功率密度 $\left(8.17 \mathrm{~W} \cdot \mathrm{g}^{-1}\right)$, 高应变 $(3.85 \%-18.6 \%)$ 的驱动。

与上述旋转驱动纤维状人工肌肉类似, 通过 客体材料的设计, 可制备具有不同刺激源响应性 的伸缩驱动纤维状人工肌肉。螺旋结构的CNT纱 线经过乙醇浸润后可保持结构完整性, 因此可通 过吸附多种溶剂产生驱动信号 ${ }^{52}$, 该结构除了由 CNT纱线构成外, 还可是CNT与石墨烯 ${ }^{33}$ 、还原氧 化石墨烯 $(\mathrm{rGO}){ }^{50}$ 或 $\mathrm{GO}^{54}$ 的复合纱线。采用硅胶作 为客体材料, 复合 CNT螺旋卷绕纱线可因吸附、脱 附丙酮实现 $2 \mathrm{MPa}$ 应力下 $50 \%$ 的可逆收缩 55 。Jin等 56 利用自合股的方式制备了具有稳定螺旋结构的人
工肌肉。该人工肌肉像天然肌肉一样, 在未发生驱 动时处于无张力状态且吸附丙酮等有机溶剂后能 够产生约 $13.3 \%$ 的收缩变形。采用亲水性高分子为 客体材料时, 复合纱线可由水或者相对湿度变化 进行驱动(图 $5 \mathrm{~g}$ ) 45,57 。

除了受电化学和溶剂吸附驱动外, 人工肌肉 还可由温差驱动。Lima等 ${ }^{23}$ 制备的两端固定, 复合 石蜡的螺旋结构 CNT纺线在脉冲时间为 $50 \mathrm{~ms}$, $18.3 \mathrm{~V} \cdot \mathrm{cm}^{-1}$ 的电压加热下, 可提升相当于自身重 量 17700 倍的重物, 产生 $3 \%$ 的收缩应变并循环运行 超过 140 万个周期。进一步增大通电电压, 减小脉 冲时间, 可优化其驱动性能。在脉冲时间为 $15 \mathrm{~ms}$, $32 \mathrm{~V} \cdot \mathrm{cm}^{-1}$ 的电压加热下, 复合纱线所提重物重量 提高至自身重量的175000倍(收缩应变 1\%)。收缩 过程做功 $0.836 \mathrm{~J} \cdot \mathrm{g}^{-1}$, 对应输出功率密度为 27.9 $\mathrm{W} \cdot \mathrm{g}^{-1}$, 是哺乳动物骨骼肌输出功率 $\left(0.323 \mathrm{~W} \cdot \mathrm{g}^{-1}\right)$ 的 85 倍。基于温差驱动发生伸缩形变的人工肌肉, 其客体材料还可扩展为弹性体和甲醇的混合物 ${ }^{58}$ 、 热塑性聚氨酯树脂 59 、环氧树脂 60 等。

在常规的主客体结构中, 客体材料的复合方 式是从内到外均匀地填充在 CNT纱线间隙中。而 Baughman教授团队 ${ }^{61}$ 研究发现, 人工肌肉在对外 输出机械能时, 纤维纱线中心部分的纤维做功甚 少, 其外层是提供机械能输出的主要部分。且无论 是基于热驱动、电化学驱动或者溶剂驱动, 所需的 刺激如热量、电荷及溶剂分子, 在纱线内部和外部 的传输均需要较长时间, 是限制人工肌肉纤维响 应速度的一大原因。因此Baughman教授团队 ${ }^{61}$ 提 出了一种新型的人工肌肉结构——鞘-芯双层结构 (图6a-e)。内芯采用加捻或螺旋卷绕的CNT纱线, 鞘层选用聚(环氧乙烷)与四氟乙烯和乙烯基醚磺 酰氟(SFVE)共聚物的混合物( $\left.\mathrm{PEO}-\mathrm{SO}_{3}\right)$, 或者弹性 聚氨酯 $(\mathrm{PU})$ 等, 所制备的PEO- $\mathrm{SO}_{3} @ \mathrm{CNT}$ 人工肌 肉在空气中通过吸收乙醇蒸汽驱动或电热致动, 最大可分别产生 $4.44 \mathrm{~W} \cdot \mathrm{g}^{-1}$ (收缩形变 $13 \%$ ) 和 2.6 $\mathrm{W} \cdot \mathrm{g}^{-1}$ (收缩形变 $\sim 8.2 \%$ ) 的平均收缩功率密度, 相 比常规的主客体复合结构, 该结构将人工肌肉的 工作能力提高了 1.7-2.15倍。Ren等62构筑了一种离 子液体填充纳米纤维鞘层的螺旋结构复合 CNT纱 线, 通过自绞合和剪切组装成电化学驱动的人工 肌肉(图6f), 该人工肌肉具有良好的稳定性及驱动 性能, 在 $0.05 \mathrm{~Hz}$ 的电压频率下, 收缩应变达到 $11.6 \%$, 与弹簧集成可制备具有抓取、保持并释放 重物能力的三爪抓手。为了使整个纱线体积都可 实现有效的驱动, Wang 等 ${ }^{63}$ 将四股 CNT纱线加捻, 设计了一种内部具有高捻度结构的人工肌肉纤 
(a)

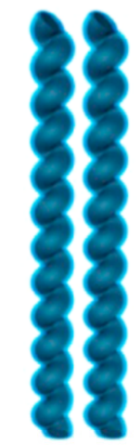

(b)

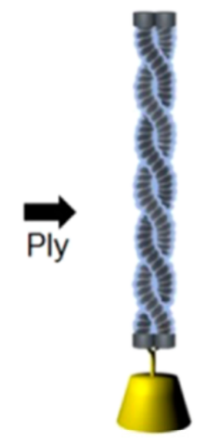

(e)

(d)

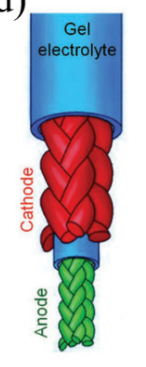

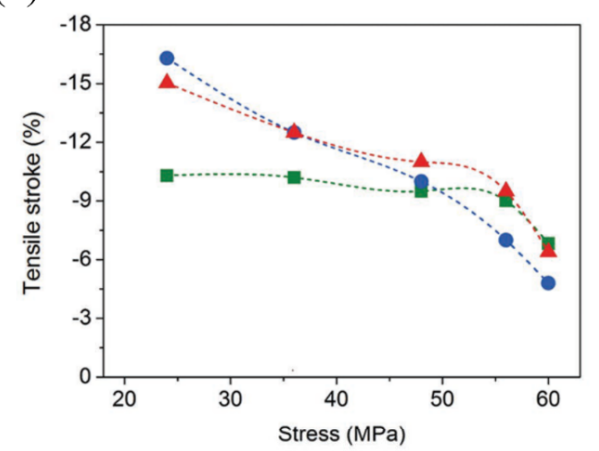

(f) (c)

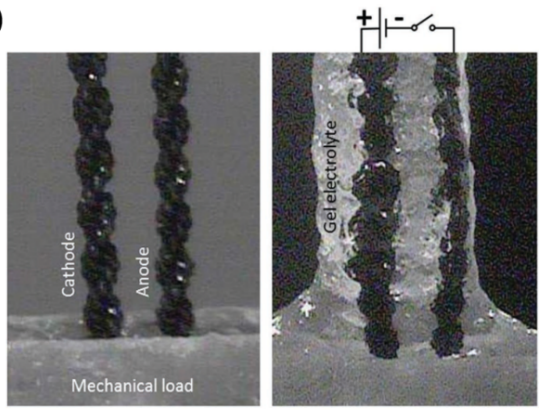

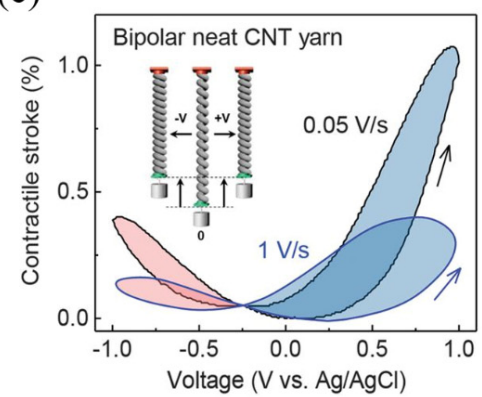

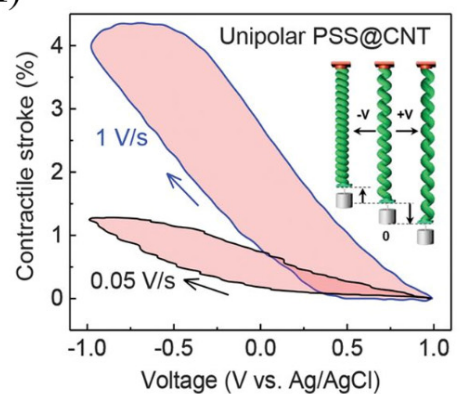

(g)

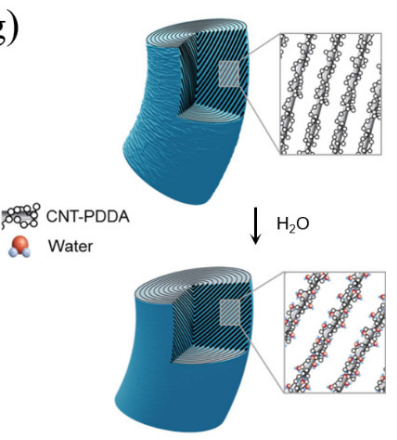

图 5 伸缩驱动的烯碳纤维人工肌肉

Fig. 5 Carbonene artificial muscle fibers with tensile actuation.

(a) Illustration of a plied, coiled yarn muscle with attached weight for tensile actuation measurements, which had been over coated with electrolyte after plying. Adapted with permission from Ref. 39. Copyright 2014, American Chemical Society. (b) The stress dependence of tensile stroke in various electrolytes. Green, blue, and red lines and data points were used in this figure to describe data for $0.2 \mathrm{M} \mathrm{TEA} \cdot \mathrm{BF}_{4}, 0.2 \mathrm{M} \mathrm{TBA} \cdot \mathrm{PF}_{6}$, and $0.2 \mathrm{M}$ THA $\cdot \mathrm{PF}_{6}$ electrolytes, respectively. (c) Optical microscope images of parallel, two-ply coiled yarns before and after coating with gel electrolyte to make a gel electrolyte muscle. (d) The structure of braided muscle. Adapted with permission from Ref. 49. Copyright 2017, WILEY-VCH. Comparisons of (e) bipolar and (f) unipolar artificial muscles. Adapted with permission from Ref. 51. Copyright 2021, AAAS. (g) Schematic illustration of the change in structure of hybrid yarn artificial muscle due to water absorption. Adapted with permission from Ref. 57. Copyright 2016, Nature Publishing Group.

维。在3.0 V电压驱动下, 液体电解质中的CNT纱 线可在 $5 \mathrm{~s}$ 内产生 $62.4 \%$ 的收缩形变, 举起相当于纱 线重量10000倍的重物。

\section{2 薄膜状烯碳人工肌肉}

Baughman教授团队 ${ }^{64}$ 发现从坚直阵列直接抽 出的 $\mathrm{CNT}$ 气凝胶膜在电刺激下可产生面内形变(图 $7 \mathrm{a})$ 。对真空中的CNT膜两端施加 $2 \mathrm{kV}$ 电压, 膜的 温度超过 $1000{ }^{\circ} \mathrm{C}$, 此时膜在长度方向发生 $1 \%$ 的收 缩, 并产生 $10 \mathrm{MPa}$ 的收缩应力, 同时宽度方向伸 长 $60 \%$ 。二维薄膜材料的面内形变往往存在变形量 小的问题, 且长度/宽度方向形变的驱动方式在实 际场景中的应用有限。而对于薄膜材料的弯曲驱 动, 其驱动形式可设计、可编程, 具有多样性。烯 碳材料由于自身优异的力学性能以及柔性特征, 是 制备薄膜材料的理想构筑基元。因此基于烯碳材 料且具有不对称结构的二维薄膜, 是一类受广泛 关注及研究的弯曲驱动器, 本节将重点讨论驱动 形式为弯曲致动的薄膜状人工肌肉。

\subsection{1 全烯碳材料的薄膜驱动器}

1999年, Baughman等 ${ }^{65}$ 首次报道了基于 SWNT 膜的电化学驱动器。当对SWNT施加电压时, 大量 电荷注入 CNT, 此时溶液中带相反电荷的离子会 吸附在 $\mathrm{CNT}$ 表面, 由于离子的注入以及离子间的 静电排斥, SWNT管发生伸长及膨胀。不同离子的 排斥力不同, 导致两侧 SWNT膜的形变不一致, 从 而整体产生弯曲驱动(图 7b, c)。Qu教授团队 66 分别 用氧等离子体和正己烷等离子体处理 $\mathrm{rGO}$ 膜两侧, 构筑了两侧分别具有亲水 (接触角约为 $15^{\circ}$ ) 和疏水 (接触角约为 $90^{\circ}$ ) 结构的不对称 $\mathrm{rGO}$ 薄膜。在水性 电解质中, 两侧电化学活性的差异导致薄膜在电 压驱动下产生不对称形变, 发生弯曲(弯曲率最大 为 $0.6 \mathrm{~cm}^{-1}$ ) (图7d, e)。Ruoff课题组 ${ }^{67}$ 用真空抽滤 方法制备了 $\mathrm{GO} / \mathrm{CNT}$ 双层膜驱动器, 由于CNT因表 面疏水对湿度不敏感，而 $\mathrm{GO}$ 的层间距可随湿度发 生变化, 即 $\mathrm{GO}$ 薄膜可吸水发生体积形变。因此复 合膜能够随湿度变化产生不同程度的弯曲。GO片 
(a)

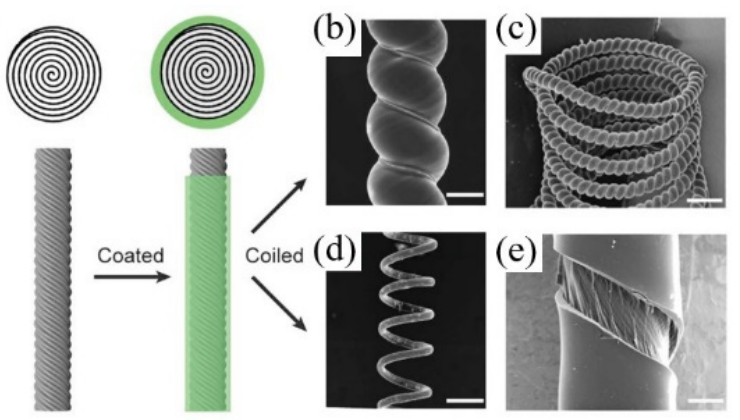

(f)

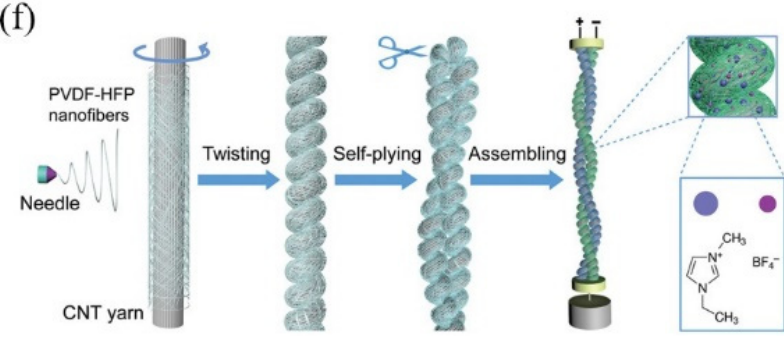

图 6 鞘-芯双层结构的烯碳人工肌肉纤维

Fig. 6 Carbonene artificial muscle fibers with sheath-core structures.

(a) Artificial muscle with sheath-core structure was fabricated by coating a twisted CNT yarn with a polymer sheath, which could be further coiled to have diverse structure as illustrated in (b-d). (e) The surface of a twisted muscle, which was broken by untwisting in liquid $\mathrm{N}_{2}$, showing the distinct boundary between sheath polymer and CNT core. Scale bars, (b) to (e), 35 200, 200 and $15 \mathrm{~mm}$, respectively. Adapted with permission from Ref. 61 .

Copyright 2019, AAAS. (f) Preparation of the solid-state electrochemical yarn muscle which integrates two yarn electrodes twisted together and separated by ionic-liquid-infiltrated PVDF-HFP nanofiber separators.

Adapted with permission from Ref. 62. Copyright 2021, Wiley-VCH.

层上的含氧官能团使其具有良好的亲水性, 其组 装体可由于片层在不同温度和湿度下发生水分的 吸脱附而产生形变, 且形变能力取决于 $\mathrm{GO}$ 片层的 含氧官能团数量 68 。一方面, 通过控制成膜过程中 不同结构 GO片层的迁移可构筑具有梯度含氧官 能团结构的 $\mathrm{GO}$ 薄膜 69 。另一方面通过直接制备或 者对 $\mathrm{GO}$ 薄膜进行单侧还原, 可得到 $\mathrm{GO} / \mathrm{rGO}$ 不对 称薄膜 ${ }^{70-73}$ 。这些内部片层具有不同化学结构的石 墨烯基薄膜, 都能在温度/湿度驱动下发生弯曲变 形。此外, Qu教授团队 ${ }^{74}$ 研究表明, 在光滑基底上 $\mathrm{GO}$ 溶液通过溶剂挥发诱导自组装所得的薄膜具 有不对称结构, 靠近基底一侧由于基底的诱导作 用表面光滑, 而与空气接触一侧在溶剂挥发的诱 导下会相对粗糙(图7f-h), 该单层薄膜同样可受湿 度驱动。

\section{2 .2 基于烯碳材料的异质双层膜驱动器}

相比于具有梯度/不对称化学结构的纯烯碳薄
膜驱动器, 异质双层膜结构的驱动器得益于两层 材料形变能力的显著差异, 可获得更高的弯曲形 变能力。烯碳材料薄膜为异质双层膜驱动器中的 一层时, 除了作为提供形变的驱动层外, 还可由于 自身的电导性、电热转化和光热转化能力赋予双 层膜电、光刺激响应能力。对于异质双层结构的薄 膜驱动器, 双层膜间的界面相互作用强弱, 决定了 双层膜结构在反复弯曲驱动过程中的稳定性, 材 料的选择和界面设计是构筑双层膜结构驱动器中 的关键问题。

$\mathrm{Qu}_{\text {教授团队 }}{ }^{75}$ 利用 $\mathrm{rGO}$ 与PPy在电解液中可受 外加电压刺激发生形变的特征, 制备了电化学驱 动的 $\mathrm{rGO} / \mathrm{PPy}$ 双层薄膜, 其弯曲率可达 $1 \mathrm{~cm}^{-1}$ 。为 了使沉积的PPy层与 $\mathrm{rGO}$ 结合的更紧密, 该工作在 电沉积前利用氧等离子体对 $\mathrm{rGO}$ 表面进行了亲水 化处理。同样的, 基于 CNT/PPy双层结构的薄膜也 具有电化学驱动性能 ${ }^{76}$ 。Shi等 ${ }^{77}$ 在 $\mathrm{GO}$ 膜上蒸镀一 层金膜, 利用金的导电性, 对驱动器通电使其温度 升高, 结合 $\mathrm{GO}$ 受热产生形变和金膜热膨胀系数低 的特征, 制备了电热响应的弯曲驱动器。进一步通 过激光加工使复合膜获得特定的形状，可构造模 仿猪笼草的双层膜驱动器。 $\mathrm{Xu}$ 等 ${ }^{78}$ 以小尺寸的 $\mathrm{GO}$ (SGO)为热致收缩层, 聚偏二氟乙烯(PVDF)为热 膨胀层, 通过刮涂法制备了双层膜驱动器。结合两 个热膨胀系数相反的材料, 提高了双层膜的热致 弯曲驱动效果。由于SGO可以对湿度、热同时发生 响应, 且具有光热效应, 因此构造的驱动器能同时 被多种刺激源(湿度、热、光)驱动, 并展示出良好 的驱动性能。综合石墨烯和特定排列 CNT 具有的 负热膨胀系数及导电性的特点, 通过复合聚二甲 基硅氧烷(PDMS)等热变形材料,可制备焦耳热响 应的弯曲驱动器, 进一步可设计出能负重行走的 软体机器人(如图7i) ${ }^{79}$, 以及抓举重物的机械爪(如 图7j) ${ }^{80,81}$ 。

\subsection{3 基于三明治结构的弯曲驱动器}

烯碳材料的二维组装体薄膜, 具有高电导率、 柔性以及耐酸碱等优点, 是一种良好的柔性集流 体以及电极材料 $30,82,83$ 。基于离子聚合物-金属复 合材料(IPMC)的人工肌肉具有三明治结构, 其由 膜状的离子聚合物骨架和镀在两侧表面的贵金属 电极组成。对IPMC薄膜施加电压时, 薄膜向阳极 弯曲产生驱动。为保证电极的导电性良好, 同时具 有化学惰性, 不被电解液腐蚀, 传统的IPMC一般 采用贵金属铂或者金作为电极, 因此驱动器造价 昂贵。金属在反复弯折后容易产生裂纹导致中间 层的电解质泄露, 降低了驱动器性能和使用寿命 
(a)

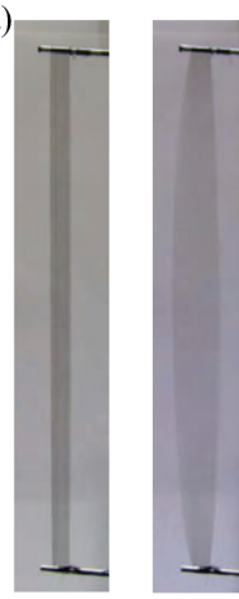

(b)

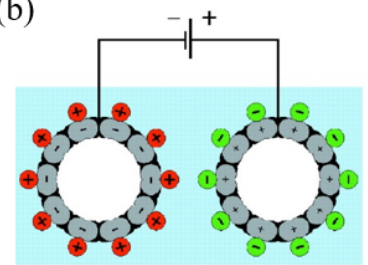

(c)

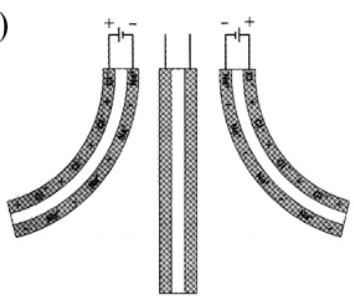

(d)

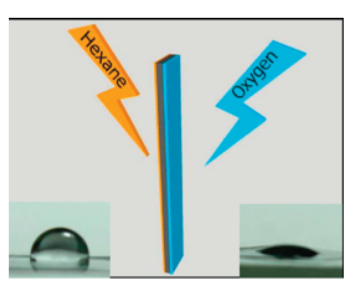

(e)

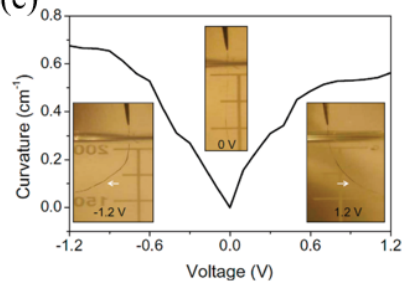

(i)

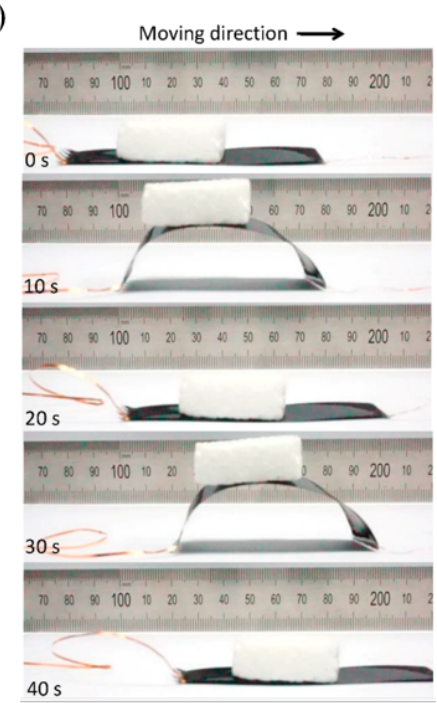

(f)
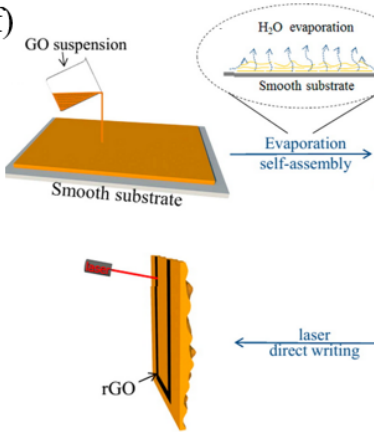
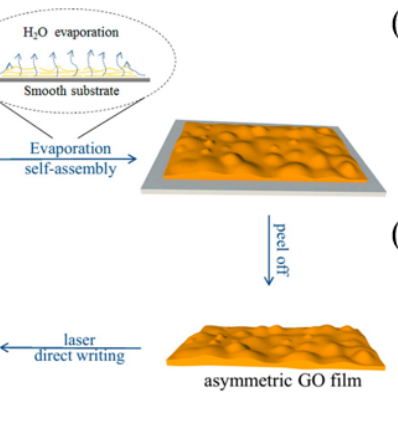

(g)

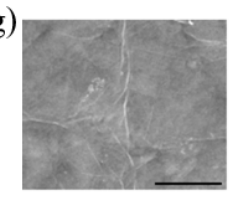

(h)
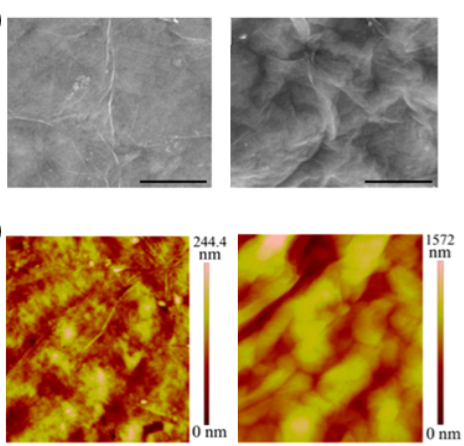

(j)

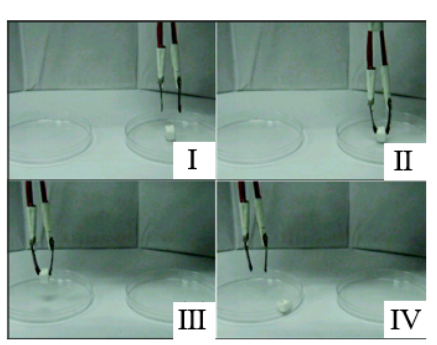

图 7 基于烯碳材料的薄膜状人工肌肉

Fig. 7 Artificial muscle films based on carbonene materials.

(a) Photograph of a rigidly end-supported 50-mm-long by 2-mm-wide CNT sheet strip (left) and CNT sheet strip expanded in width by applying $5 \mathrm{kV}$ with respect to ground (right). Adapted with permission from Ref. 64. Copyright 2009, AAAS. (b) Schematic illustration of charge injection in a nanotube-based electromechanical actuator. (c) Schematic edge-view of an actuator operated in aqueous $\mathrm{NaCl}$, which consists of two strips of SWNTs (shaded) that are laminated together with an intermediate layer of double-sided Scotch tape (white). Adapted with permission from Ref. 65. Copyright 1999, AAAS.

(d) Schematic illustration of asymmetric plasma treatments of the graphene film with hexane and oxygen, and the wettability of corresponding surface.

(e) Curvature change of the actuator as a function of applied CV potential. Adapted with permission from Ref. 66. Copyright 2010, American Chemical

Society. (f) Fabrication scheme of the GO film responsive actuator with asymmetric structure. (g) SEM and (h) AFM images of smooth side (left) and rough side (right) of the GO film, respectively. Scale bar of SEM image: $5 \mu \mathrm{m}$. Adapted with permission from Ref. 74. Copyright 2016, American

Chemical Society. (i) Optical photo series showing the moving process of the weightlifting walking robot. Adapted with permission from

Ref. 79. Copyright 2015, American Chemical Society. (j) A demonstration of a gripper consisting of two actuators manipulating a small object.

Adapted with permission from Ref. 81. Copyright 2011, American Chemical Society.

(图8a) ${ }^{84}$ 。烯碳材料的出现使IPMC人工肌肉中的 金属电极有了理想的替代品, 并促使其获得长足 的发展 ${ }^{16,84-88}$ 。

$\mathrm{Li}$ 等 ${ }^{85}$ 通过热压的方法, 构筑了两侧为自支撑 SWNT薄膜, 中间为壳聚糖/聚离子液体电解质的 三明治结构驱动器。由于SWNT的多孔结构及其与 其他组分间良好的相容性, SWNT电极与中间电解 液层结合紧密, 且SWNT自身优异的力学性能也有 效提高了复合驱动器的强度与模量。SWNT的高电 导率和高比较面积, 加快了电极之间的电荷注入 和离子迁移速度, 赋予驱动器大而快的弯曲响应
形变。该驱动器可在 $19 \mathrm{~ms}$ 内受激发生形变, 使用 频率范围在几十到数百赫兹。

Im 等 ${ }^{86}$ 以Nafion膜为中间绝缘层, 两侧通过物 理接触结合石墨烯膜，制备的三明治结构薄膜可 获得弯曲率最高为 $0.3 \mathrm{~cm}^{-1}$ 的弯曲驱动效果。薄膜 中石墨烯与中间绝缘层结合力弱, 因此其三明治 结构易由于反复的弯曲变形而破坏, 降低了驱动 器的循环使用寿命。 $\mathrm{Kim}^{\text {等 }}{ }^{84}$ 用激光处理 $\mathrm{rGO}$ 的一 面使其粗糙化，增强其与聚合物中间层的结合力 (图8b), 制备得到的弯曲驱动器可以在 $4.5 \mathrm{~V}$ 电压 下稳定循环驱动 360 次, 且性能无明显下降。除了 
(a) Conventional lonic Polymer-Metal Composite (IPMC) Actuator

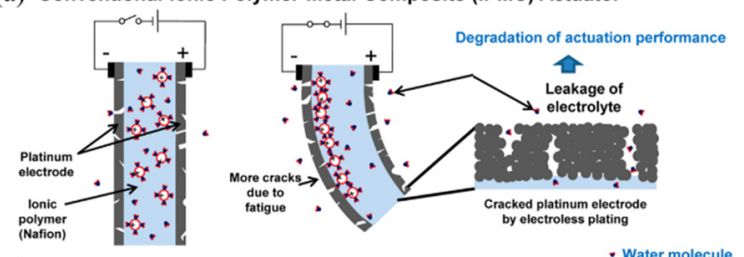

(b) Novel lonic Polymer-Graphene Composite (IPGC) Actuator Hydrated cation

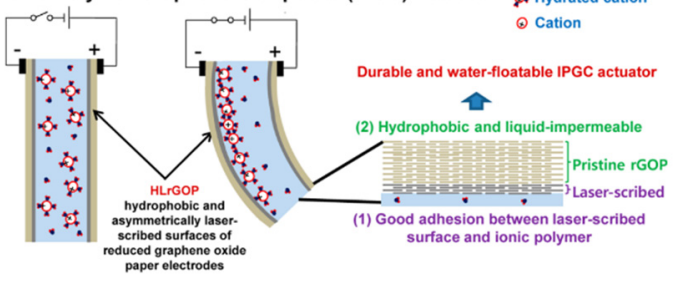

图 8 IPMC 与 IPGC 的结构

Fig. 8 Schematic representations of the structures of IPMC and IPGC.

(a) Liquid-permeable IPMC actuator with cracks in metal electrodes.

(b) Durable and water-floatable IPGC actuator with graphene films as electrodes. Adapted with permission from Ref. 84. Copyright 2014,

American Chemical Society.

用于替换IPMC中的金属电极以外，烯碳薄膜也可 用作驱动介电弹性体发生形变的电极材料 ${ }^{89,90}$ 。

\section{3 烯碳材料在人工肌肉中的复合功能化 应用}

烯碳材料由于具有高强度、高模量及高比表 面积的特征，且可利用多种手段实现表面修饰从 而为烯碳材料与其他材料提供丰富的相互作用形 式(离子键、氢键、 $\pi-\pi$ 相互作用以及共价键等单一 或多种协同作用)。因此, 烯碳材料可用作复合材 料中的增强相, 通过与基体间的相互作用, 耗散应 力从而达到复合增强的目的 ${ }^{91}$ 。对于其他力学性能 较弱的人工肌肉材料, 可通过复合烯碳材料提高 力学性能以获得稳定的驱动结构 35 。此外, 得益于 烯碳材料的高导电性和光热、电热转化能力, 复合 了烯碳材料的人工肌肉, 可由单一刺激源扩展为 多重刺激响应, 并且具有可编程的响应形变及感 知能力。

\section{1 增强及扩展刺激响应功能}

形状记忆材料, 例如形状记忆合金、形状记忆 高分子以及形状记忆陶瓷等, 因具有形状记忆效 应(在外界刺激下恢复为变形前的形状), 可用作人 工肌肉材料。Yuan等 ${ }^{92}$ 对处于玻璃化转变温度以 上的PVA纤维实施加捻, 随后降温将捻应力固定。 当再次加热到玻璃化温度以上, 就可实现解捻。该 工作结合PVA的形状记忆效应和捻曲技术, 制备
了扭矩平衡的热驱动转动人工肌肉。通过复合 SWNT和GO, 纤维室温下的杨氏模量分别增加至 13.5 和 $12.5 \mathrm{GPa}$ (纯的PVA纤维剪切模量: $4.9 \mathrm{GPa}$ ) (图9a), 获得了更高的驱动功率密度和驱动扭矩。 PVA-GO和PVA-SWNT纤维人工肌肉可分别产生 $\sim 0.27$ 和 $0.12 \mu \mathrm{N} \cdot \mathrm{m}$ 的扭矩, 2.766 和 $1.115 \mathrm{~J} \cdot \mathrm{g}^{-1}$ 的能 量密度, 远高于纯的PVA人工肌肉(扭矩: 0.11 $\mu \mathrm{N} \cdot \mathrm{m}$; 能量密度: $0.925 \mathrm{~J} \cdot \mathrm{g}^{-1}$ ) (图9b)。由于 $\mathrm{GO}$ 具 有二维片层结构, 因此相比于SWNT, 对PVA扭转 性能的改善更为明显。

介电弹性体是一种介电常数高且具有弹性的 聚合物。当向其薄膜两侧施加电压时, 带相反电荷 电极间的相互吸引力使薄膜压缩, 而同一侧由于 相同电荷的排斥力使薄膜伸长, 即发生电驱动。通 过复合烯碳材料, 可使介电弹性体的机电性能发 生变化 ${ }^{93,94}$ 。例如Wang 等 ${ }^{93}$ 将多壁CNT填充到介电 弹性体中, 获得了力学性能和导电性得到提高的 复合材料, 相比与纯的介电弹性体驱动器, 复合材 料驱动器可在更低电压刺激下产生更高的形变 (28\%) (图9c)。

刺激响应水凝胶中交联的高分子网络可在外 界刺激下 (热、 $\mathrm{pH}$ 、光、电场及生物分子等)可逆吸 收/排除水分发生溶胀/收缩从而产生体积形变, 是 一种典型的软体驱动器。虽然水凝胶对外输出 的驱动力非常小, 但巨大的体积形变是其优势。 PNIPAm 是具有良好热响应的水凝胶, 但透明的特 性使其对光无响应, 而光驱动器具有可以远程操 控等优点。将GO或 $\mathrm{rGO}$ 与PNIPAm复合, 既能增强 水凝机械强度, 又可利用 $\mathrm{GO} / \mathrm{rGO}$ 的光热转化效应 实现水凝胶的光热驱动。基于复合结构的水凝胶 具有光致弯曲的性能, 例如 GO/PNIPAm 和 PNIPAm双层结构水凝胶受光照后在约 $75 \mathrm{~s}$ 内发生 弯曲驱动, 具有梯度 GO含量的 GO/PNIPAm水凝胶 弯曲率最高能到 $2.4 \mathrm{~cm}^{-1}$, 以及 $\mathrm{rGO} / \mathrm{PNIPAm}$ 和聚 丙烯酰胺组成的异质双层结构水凝胶在光强为 $63 \mathrm{~mW} \cdot \mathrm{cm}^{-2}$ 时, 弯曲率可达 $120 \mathrm{~cm}^{-1}$, 响应时间 为 $30 \mathrm{~s}^{95}$ 。半晶态聚合物在受热时, 内部结晶区域 会发生体积膨胀而无定形的分子链则发生长度方 向的收缩。因此高度取向的半晶态聚合物纤维, 例 如聚乙烯和尼龙纤维, 受热时会发生长度收缩并 伴随着直径膨胀。Baughman教授团队 ${ }^{48}$ 将捻曲技 术应用在尼龙 6,6 纤维上, 制备了具有螺旋结构的 尼龙纤维人工肌肉。尼龙纤维受热发生形变可使 加捻结构解捻, 因此该纤维可在温度刺激下产生 $49 \%$ 的驱动量, 输出功率可达 $27.1 \mathrm{~W} \cdot \mathrm{g}^{-1}$ 。若将 CNT 薄膜缠绕在纤维表面, 利用CNT的导电性, 可通过 
(a)

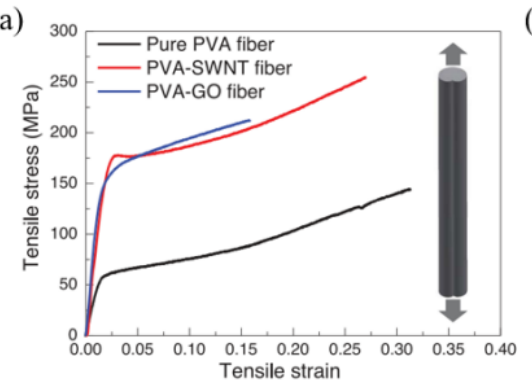

(b)

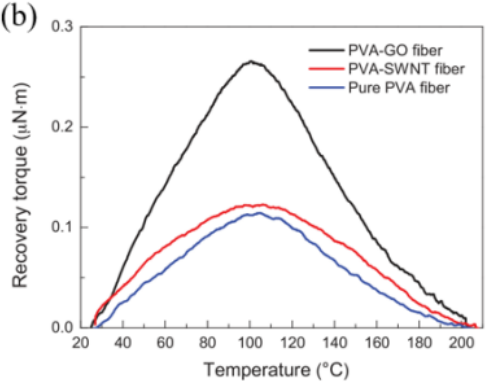

(c)

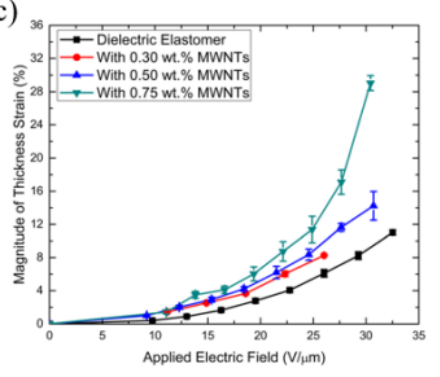

(d)

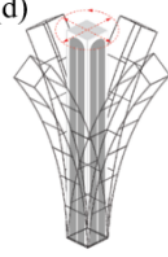

(e)

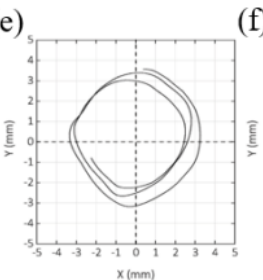

(f)

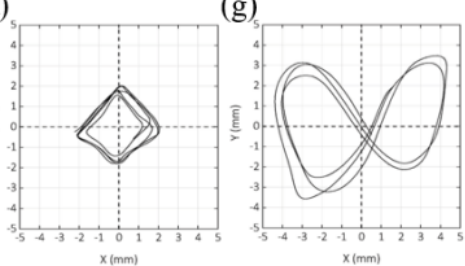

(h)
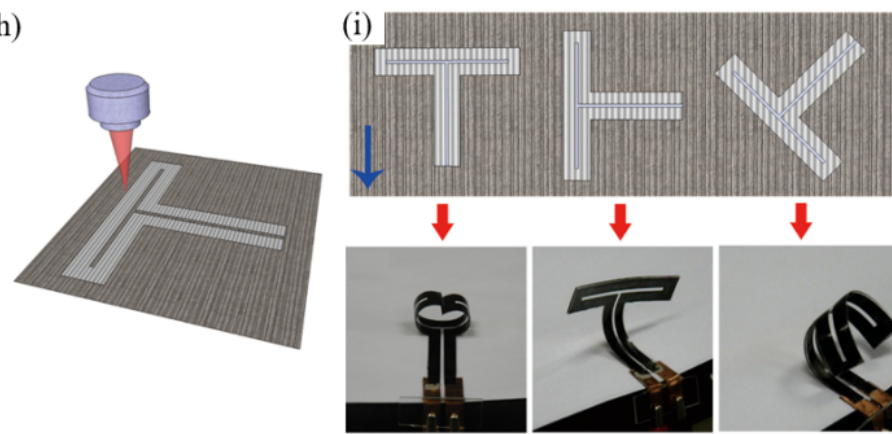

(j)

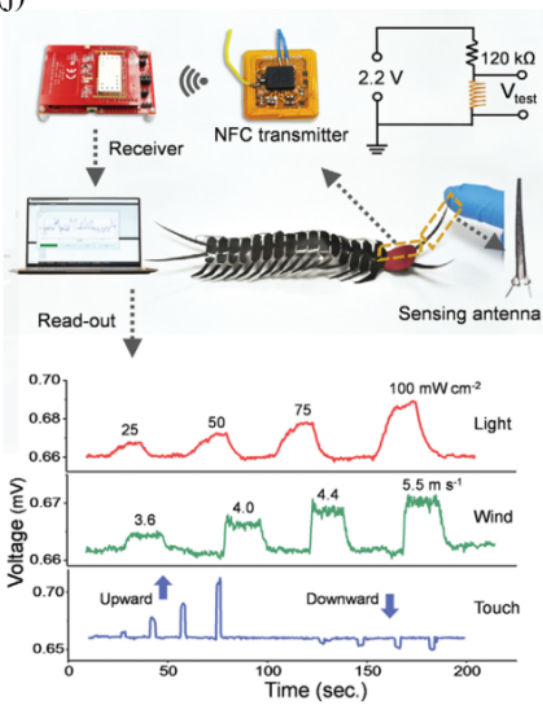

图 9 用于增强与赋能人工肌肉的烯碳材料

Fig. 9 Mechanical enhancements and multi-functionalization of artificial muscles by carbonene materials.

(a) Enhancement on tensile stress and modulus of PVA artificial muscle fibers by compositing GO and CNT. (b) Recovery torque generated by the coiled fibers that have been quenched without being hooked when they are reheated. Adapted with permission from Ref. 92. Copyright 2019, AAAS.

(c) Measured and computed thickness strain of dielectric elastomer nanocomposites under applied electric fields. Adapted with permission from Ref. 93.

Copyright 2017, AIP. (d) Schematic of the multidirectional actuator. (e-g) The coordinate of the tip of the actuator by applying the following inputs (left to right), $X$-Ch: $V_{x}=V_{x o} \sin (\omega \cdot t), Y-C h: V_{y}=\mathrm{V}_{\mathrm{yo}} \cos (\omega \cdot t) ; X-C h: V_{x}=V_{x o} \operatorname{tringl}(\omega \cdot t), Y-C h: V_{y}=V_{y o} \operatorname{tringle}(\omega \cdot t+\pi / 2) ; X-C h: V_{x}=V_{x o} \sin (2 \omega \cdot t)$, Y-Ch: $V_{y}=V_{y o} \cos (\omega \cdot t)$. Adapted with permission from Ref. 97. Copyright 2016, WILEY. (h) Schematic of graphic design and laser cutting on BP to make a

T-shaped conductive band. (i) Three different ways of processing T-shaped BP electrodes and corresponding actuations. Adapted with permission from Ref. 98. Copyright 2015, American Chemical Society. (j) Wireless antenna sensing of light illumination intensity, wind speed and touch via near-field communication. Adapted with permission from Ref. 106. Copyright 2020, WILEY-VCH.

焦耳热驱动纤维变形。

\section{2 可编程的响应变形}

除了前述具有不对称结构的人工肌肉可受刺 激产生弯曲驱动外, 对结构均一的材料施加不对 称刺激, 也可实现弯曲驱动。通过合理设计材料结 构, 制备具有更复杂响应变形的人工肌肉才能满 足多场景下的先进应用需求 ${ }^{96}$ 。由于烯碳材料的可 加工性，通过简单的方法即可实现其在刺激响应 材料中的图案化, 从而利用其产生局部刺激, 最终 实现人工肌肉可编程的响应形变。实际上, 烯碳材 料在人工肌肉中的这一类应用, 也是基于其电热/ 光热转化效应。
Mirvakili等 ${ }^{97}$ 采用浸渍涂覆的方法在梁状的 尼龙表面覆盖了含有石墨烯的光热转化功能涂 料, 并利用高功率的激光二极管实现对尼龙梁的 光热驱动。通过对梁的单一或者多个侧面施加刺 激, 可实现人工肌肉的多方向驱动(图 $9 \mathrm{~d}-\mathrm{g}$ )。 Li 等 98 从 CNT坚直阵列中抽出气凝胶薄膜, 并经过双 辊压机获得具有高度各向异性的巴基纸(Bucky paper, BP)。利用激光对CNT巴基纸进行图案化加 工, 可得到具有特殊形状且面内CNT沿着特定方 向严格排列的巴基纸(图9h)。以其为柔性电极, 与 PDMS复合, 制备双层结构的驱动器。由于巴基纸 在平行于 $\mathrm{CNT}$ 和垂直于 $\mathrm{CNT}$ 两个面内方向上的导 
电性存在巨大差异。经过图案化后巴基纸面内可 产生不同的电热效应, 从而使PDMS层部分区域受 热发生形变。通过精巧的设计, 该驱动器可产生大 的弯曲形变 $\left(>180^{\circ}\right)$, 螺旋卷曲 $\left(\sim 630^{\circ}\right)$ (图9i), 甚 至实现模仿手指的仿生驱动。利用激光诱导生成 石墨烯的方法实现图案化 ${ }^{99-101}$, 或者合理设计双 层结构驱动器中石墨烯层的位置 ${ }^{102,103}$, 都可对人 工肌肉材料的形变进行编程设计。

\section{3 传感功能}

对于柔性和人工智能机器人而言, 装配准确 的具有实施运动感知能力的肌肉材料, 对其实现 对环境的自主响应具有重要意义。因此开发集驱 动和传感一体的人工肌肉是该领域的必然趋势。

人工肌肉的驱动过程总是伴随着体积的变化, 若 该形变可改变其中烯碳材料构建的导电网络通 路, 在恒压电源下, 由于烯碳材料电阻的变化, 材 料可输出不同的电流信号。因此烯碳材料还可在 人工肌肉中起到传感的功能 ${ }^{104-107}$ 。

Wang 等 ${ }^{106}$ 以聚多巴胺还原的氧化石墨烯 (PDG) 和PVDF制备了双层膜结构的驱动器, 并在 PVDF另一侧3D打印了基于石墨-CNT的U形电路。 由 PDG 与 PVDF层构成的驱动器除了具有热响应 功能外, 还可通过PDG层的光热转换功能实现光 响应驱动。当温度发生变化时, PVDF中由反式 $\beta$ 相 排列的聚合物链产生的永久偶极子的取向随之变 化, 并在PVDF的厚度方向上产生热电势, 因此 PVDF层可起到自供电的温度传感功能。而U型的 石墨-CNT电路, 其内阻随PVDF形变发生变化, 可 作为应变传感器。集光、热响应和温度、形变传感 为一体的薄膜状驱动器, 由于结构柔性和可加工 性的特征, 通过引入可编程的折纸技术, 可进一步 设计制造出具有综合感知和运动能力的薄膜机器 人(图9j)。除了传感体系的应变外, 烯碳材料还可 用作温度传感部件, 例如复合了甲基纤维素的 CNT膜具有负的电阻温度系数 $\left(-3807.7 \mathrm{ppm} \cdot \mathrm{K}^{-1}\right)$, 因此在双层膜驱动器中还可用作温度传感层 ${ }^{107}$ 。

\section{4 总结与展望}

烯碳材料在人工肌肉领域的应用, 概括而言 均是从其独特的理化特性出发, 作为人工肌肉中 的结构、功能性材料的应用。以烯碳材料为基本组 成单元的人工肌肉, 宏观表现形态可为一维纤维 和二维薄膜。由于形态上更接近生物肌肉中的肌 原纤维, 纤维状人工肌肉在仿生驱动器中更具有 优势。而基于烯碳材料的纤维状人工肌肉, 可通过 组装结构的设计, 实现高效的弯曲、旋转和伸缩驱
动。特别地, 具有加捻和螺旋结构烯碳纤维的制 备, 为推动仿生肌肉纤维的发展起到了重要作用。 基于烯碳材料的二维薄膜状人工肌肉, 驱动形式 以弯曲驱动为主。在这一类集响应、驱动及结构为 一体的人工肌肉中, 烯碳材料和整体不对称结构 中的结构设计、客体材料和异质材料的种类选择 是其设计与制备中的关键问题。当烯碳材料在纳 米复合人工肌肉材料中发挥功能性应用时, 烯碳 材料可作为增强赋能相, 通过优化材料的力学、电 学特性以提升人工肌肉的驱动性能、扩展响应刺 激种类、响应形变形式以及赋予其传感功能。

虽然烯碳材料在人工肌肉领域的应用已获得 了长足的发展, 并取得了诸多优异的性能。但其中 仍有一些亟待解决的问题: (1)烯碳材料的成本问 题限制了相关人工肌肉材料的应用, 因此需开发 高效、便捷的生产和组装方法, 以用于烯碳材料及 其人工肌肉的制备。(2)能量转换效率低是人工肌 肉领域的一个痛点问题。目前, 大多数的人工肌肉 的能量转换效率仍然较低, 无法与传统的电机或 热机的能量转换效率相比。因此发展具有高能量 转换效率的人工肌肉是重要的研究方向。(3)人工 肌肉的输出力小也是限制其实际应用的一个重要 的因素。而输出力的大小又受到人工肌肉的结构 以及模量等因素影响, 发展大输出力的人工肌肉 是其迈向实际应用的关键步骤。(4)人工肌肉的真 正应用, 是多学科交叉研究后的成果。除了人工肌 肉材料的制备外, 人工肌肉在智能驱动系统中的 装配, 以及相应的驱动精确控制与反馈系统的设 计, 还需材料与机械工程、信息科学等领域知识的 互相交叉融合。在实际的装配与应用中发现问题, 从而对材料设计提出新要求, 以催生人工肌肉材 料的新发展。

烯碳材料由于自身优异的力学性能、高比表 面积、柔性及可加工的特性, 可构筑成多维的组装 材料, 而突出的电学、热学和光学性能, 又可赋予 其组装材料多功能特性。因此烯碳材料是结构一功 能一体化材料的优秀构筑基元。诸如柔性外骨骼、 仿真机器人、变翼飞行器等未来先进智能驱动系 统的发展, 要求人工肌肉除了单一的致动功能外, 还需要兼具轻质、柔性等结构特征, 并且集传感、 信号传输、控制等功能于一体, 这进一步激发了多 功能驱动的需求。从烯碳材料的结构与性能出发, 探索全新的组装方式, 开发具有高效驱动性能的 组装结构, 匹配各异的驱动形式与复合材料以寻 求最优解, 并结合烯碳材料的功能特性, 有望设计 具有结构-功能-智能一体化的人工肌肉。 


\section{References}

(1) Mirvakili, S. M.; Hunter, I. W. Adv. Mater. 2018, 30, 1704407. doi: 10.1002/adma.201704407

(2) Uchino, K. Advanced Piezoelectric Materials: Science and Technology, Woodhead Publishing Limited: Cambridge, UK; 2010.

(3) Wang, J.; Gao, D.; Lee, P. S. Adv. Mater. 2021, 33, e2003088. doi: 10.1002/adma.202003088

(4) Zou, M.; Li, S.; Hu, X.; Leng, X.; Wang, R.; Zhou, X.; Liu, Z. F. Adv. Funct. Mater. 2021, 2007437. doi: 10.1002/adfm.202007437

(5) Foroughi, J.; Spinks, G. Nanoscale Adv. 2019, 1, 4592. doi: $10.1039 / \mathrm{c} 9 \mathrm{na} 00038 \mathrm{k}$

(6) Wang, W.; Ahn, S. H. Soft Rob. 2017, 4, 379. doi: 10.1089 /soro.2016.0081

(7) Chen, Y.; Chen, C.; Rehman, H. U.; Zheng, X.; Li, H.; Liu, H.; Hedenqvist, M. S. Molecules 2020, 25, 4246 doi: 10.3390/molecules 25184246

(8) Qiu, Y.; Zhang, E.; Plamthottam, R.; Pei, Q. Acc. Chem. Res. 2019, 52, 316. doi: 10.1021/acs.accounts. 8 b00516

(9) Chen, Z. Robot. Biomim. 2017, 4, 24. doi: 10.1186/s40638-017-0081-3

(10) Smela, E. Adv. Mater. 2003, 15, 481. doi: 10.1002/adma.200390113

(11) Mirfakhrai, T.; Madden, J. D. W.; Baughman, R. H. Mater. Today 2007, 10, 30. doi: 10.1016/s1369-7021(07)70048-2

(12) Yin, Z.; Shi, S.; Liang, X.; Zhang, M.; Zheng, Q.; Zhang, Y. Adv. Fiber Mater. 2019, 1, 197. doi: 10.1007/s42765-019-00021-y

(13) Jia, T.; Wang, Y.; Dou, Y.; Li, Y.; de Andrade, M. J.; Wang, R.; Fang, S.; Li, J.; Yu, Z.; Qiao, R.; et al. Adv. Funct. Mater. 2019, 29 , 1808241. doi: 10.1002/adfm.201808241

(14) Wang, Y.; Wang, Z.; Lu, Z.; Jung de Andrade, M.; Fang, S.; Zhang, Z.; Wu, J.; Baughman, R. H. ACS Appl. Mater. Interfaces 2021, 13 , 6642. doi: 10.1021/acsami.0c20456

(15) Wang, Y. L.; Di, J. T.; Li, Q. W. Mater. Rep. 2021, 35, 1183. [王玉莲, 邸江涛, 李清文. 材料导报, 2021, 35, 1183.] doi: $10.11896 /$ cldb.20030153

(16) Kong, L.; Chen, W. Adv. Mater. 2014, 26, 1025. doi: 10.1002/adma.201303432

(17) Foroughi, J.; Spinks, G. M.; Wallace, G. G.; Oh, J.; Kozlov, M. E.; Fang, S. L.; Mirfakhrai, T.; Madden, J. D. W.; Shin, M. K.; Kim, S. J.; et al. Science 2011, 334, 494. doi: 10.1126/science.1211220

(18) Zhang, S. C.; Zhang, N.; Zhang, J. Acta Phys. -Chim. Sin. 2020, 36, 1907021. [张树辰, 张娜, 张锦. 物理化学学报, 2020, 36, 1907021.] doi: 10.3866/PKU.WHXB201907021

(19) Plisko, T. V.; Bildyukevich, A. V. Colloid. Polym. Sci. 2014, 292 2571. doi: 10.1007/s00396-014-3305-x

(20) Jian, M. Q.; Zhang, Y. Y.; Liu, Z. F. Acta Phys. -Chim. Sin. 2022, 38, 2007093. [寒木强, 张荣荣, 刘忠范. 物理化学学报, 2022, 38, 2007093.] doi: 10.3866/PKU.WHXB202007093
(21) Stoychev, G. V.; Ionov, L. ACS Appl. Mater. Interfaces 2016, 8, 24281. doi: 10.1021/acsami.6b07374

(22) Di, J.; Zhang, X.; Yong, Z.; Zhang, Y.; Li, D.; Li, R.; Li, Q. Adv. Mater. 2016, 28, 10529. doi: 10.1002/adma.201601186

(23) Lima, M. D.; Li, N.; Jung de Andrade, M.; Fang, S.; Oh, J.; Spinks, G. M.; Kozlov, M. E.; Haines, C. S.; Suh, D.; Foroughi, J.; et al. Science 2012, 338, 928. doi: 10.1126/science. 1226762

(24) Jiang, K. L.; Li, Q. Q.; Fan, S. S. Nature 2002, 419, 801. doi: $10.1038 / 419801 \mathrm{a}$

(25) Zhang, M.; Atkinson, K. R.; Baughman, R. H. Science 2004, 306, 1358. doi: 10.1126/science. 1104276

(26) Zhang, X.; Lu, W.; Zhou, G.; Li, Q. Adv. Mater. 2020, 32, 1902028. doi: 10.1002/adma.201902028

(27) Xu, Z.; Gao, C. Nat. Commun. 2011, 2, 571. doi: $10.1038 /$ ncomms 1583

(28) Xia, Z.; Shao, Y. L. Acta Phys. -Chim. Sin. 2022, 38, 2103046. [夏洲, 邵元龙. 物理化学学报, 2022, 38, 2103046.] doi: 10.3866/PKU.WHXB202103046

(29) Cheng, H.; Hu, Y.; Zhao, F.; Dong, Z.; Wang, Y.; Chen, N.; Zhang, Z.; Qu, L. Adv. Mater. 2014, 26, 2909. doi: 10.1002/adma.201305708

(30) Janas, D.; Koziol, K. K. Nanoscale 2016, 8, 19475. doi: $10.1039 / \mathrm{c} 6 \mathrm{nr} 07549 \mathrm{e}$

(31) Guo, S.; Dong, S. Chem. Soc. Rev. 2011, 40, 2644. doi: $10.1039 / \mathrm{c} 0 \operatorname{cs} 00079 \mathrm{e}$

(32) Munoz, E.; Dalton, A. B.; Collins, S.; Kozlov, M.; Razal, J.; Coleman, J. N.; Kim, B. G.; Ebron, V. H.; Selvidge, M.; Ferraris, J. P.; et al. Adv. Eng. Mater. 2004, 6, 801. doi: 10.1002/adem.200400092

(33) Shin, S. R.; Lee, C. K.; So, I.; Jeon, J. H.; Kang, T. M.; Kee, C.; Kim, S. I.; Spinks, G. M.; Wallace, G. G.; Kim, S. J. Adv. Mater. 2008, 20 , 466. doi: 10.1002/adma.200701102

(34) Lee, S. H.; Lee, C. K.; Shin, S. R.; Gu, B. K.; Kim, S. I.; Kang, T. M.; Kim, S. J. Sens. Actuators B-Chem. 2010, 145, 89. doi: 10.1016/j.snb.2009.11.043

(35) Spinks, G. M.; Mottaghitalab, V.; Bahrami-Saniani, M.; Whitten, P. G.; Wallace, G. G. Adv. Mater. 2006, 18, 637. doi: 10.1002/adma.200502366

(36) Plaado, M.; Kaasik, F.; Valner, R.; Lust, E.; Saar, R.; Saal, K.; Peikolainen, A. L.; Aabloo, A.; Kiefer, R. Carbon 2015, 94, 911. doi: 10.1016/j.carbon.2015.07.077

(37) Cheng, H.; Liu, J.; Zhao, Y.; Hu, C.; Zhang, Z.; Chen, N.; Jiang, L.; Qu, L. Angew. Chem. Int. Ed. 2013, 52, 10482. doi: 10.1002/anie.201304358

(38) Wang, Y.; Bian, K.; Hu, C.; Zhang, Z.; Chen, N.; Zhang, H.; Qu, L. Electrochem. Commun. 2013, 35, 49. doi: 10.1016/j.elecom.2013.07.044 
(39) Lee, J. A.; Kim, Y. T.; Spinks, G. M.; Suh, D.; Lepro, X.; Lima, M. D.; Baughman, R. H.; Kim, S. J. Nano Lett. 2014, 14, 2664. doi: $10.1021 / \mathrm{nl} 500526 \mathrm{r}$

(40) He, S.; Chen, P.; Qiu, L.; Wang, B.; Sun, X.; Xu, Y.; Peng, H. Angew. Chem. Int. Ed. 2015, 54, 14880. doi: 10.1002/anie.201507108

(41) Chun, K. Y.; Hyeong Kim, S.; Kyoon Shin, M.; Hoon Kwon, C.; Park, J.; Tae Kim, Y.; Spinks, G. M.; Lima, M. D.; Haines, C. S.; Baughman, R. H.; et al. Nat. Commun. 2014, 5, 3322. doi: $10.1038 /$ ncomms 4322

(42) Shi, Q.; Li, J.; Hou, C.; Shao, Y.; Zhang, Q.; Li, Y.; Wang, H. Chem. Commun. 2017, 53, 11118. doi: 10.1039/c7cc03408c

(43) Wang, W.; Xiang, C.; Sun, D.; Li, M.; Yan, K.; Wang, D. ACS Appl. Mater. Interfaces 2019, 11, 21926. doi: 10.1021/acsami.9b05136

(44) Lee, S. H.; Kim, T. H.; Lima, M. D.; Baughman, R. H.; Kim, S. J. Nanoscale 2016, 8, 3248. doi: 10.1039/c5nr07195j

(45) Gu, X.; Fan, Q.; Yang, F.; Cai, L.; Zhang, N.; Zhou, W.; Zhou, W.; Xie, S. Nanoscale 2016, 8, 17881. doi: 10.1039/c6nr06185k

(46) Kim, H.; Moon, J. H.; Mun, T. J.; Park, T. G.; Spinks, G. M.; Wallace, G. G.; Kim, S. J. ACS Appl. Mater. Interfaces 2018, 10, 32760. doi: $10.1021 /$ acsami.8b12426

(47) Lima, M. D.; Fang, S. L.; Lepro, X.; Lewis, C.; Ovalle-Robles, R.; Carretero-Gonzalez, J.; Castillo-Martinez, E.; Kozlov, M. E.; Oh, J. Y.; Rawat, N.; et al. Science 2011, 331, 51. doi: 10.1126/science.1195912

(48) Haines, C. S.; Lima, M. D.; Li, N.; Spinks, G. M.; Foroughi, J.; Madden, J. D. W.; Kim, S. H.; Fang, S.; de Andrade, M. J.; Goktepe, F.; et al. Science 2014, 343, 868. doi: 10.1126/science.1246906

(49) Lee, J. A.; Li, N.; Haines, C. S.; Kim, K. J.; Lepro, X.; OvalleRobles, R.; Kim, S. J.; Baughman, R. H. Adv. Mater. 2017, 29 , 1700870. doi: 10.1002/adma.201700870

(50) Qiao, J.; Di, J.; Zhou, S.; Jin, K.; Zeng, S.; Li, N.; Fang, S.; Song, Y.; Li, M.; Baughman, R. H.; Li, Q. Small 2018, 14, 1801883. doi: $10.1002 / \mathrm{smll} .201801883$

(51) Chu, H.; Hu, X.; Wang, Z.; Mu, J.; Li, N.; Zhou, X.; Fang, S.; Haines, C. S.; Park, J. W.; Qin, S.; et al. Science 2021, 371, 494. doi: $10.1126 /$ science.abc4538

(52) Chen, P. N.; Xu, Y. F.; He, S. S.; Sun, X. M.; Pan, S. W.; Deng, J.; Chen, D. Y.; Peng, H. S. Nat. Nanotechnol. 2015, 10, 1077. doi: $10.1038 /$ nnano. 2015.198

(53) Hyeon, J. S.; Park, J. W.; Baughman, R. H.; Kim, S. J. Sens Actuators B-Chem. 2019, 286, 237. doi: 10.1016/j.snb.2019.01.140

(54) Sun, Y.; Wang, Y.; Hua, C.; Ge, Y.; Hou, S.; Shang, Y.; Cao, A. Carbon 2018, 132, 394. doi: 10.1016/j.carbon.2018.02.086

(55) Lima, M. D.; Hussain, M. W.; Spinks, G. M.; Naficy, S.; Hagenasr, D.; Bykova, J. S.; Tolly, D.; Baughman, R. H. Small 2015, 11, 3113. doi: 10.1002/smll.201500424

(56) Jin, K.; Zhang, S.; Zhou, S.; Qiao, J.; Song, Y.; Di, J.; Zhang, D.; Li,
Q. Nanoscale 2018, 10, 8180. doi: 10.1039/c8nr01300d

(57) Kim, S. H.; Kwon, C. H.; Park, K.; Mun, T. J.; Lepro, X.; Baughman, R. H.; Spinks, G. M.; Kim, S. J. Sci. Rep. 2016, 6, 23016. doi: $10.1038 /$ srep23016

(58) Jeong, J. H.; Mun, T. J.; Kim, H.; Moon, J. H.; Lee, D. W.; Baughman, R. H.; Kim, S. J. Nanoscale Adv. 2019, 1, 965. doi: $10.1039 / \mathrm{c} 8 \mathrm{na} 00204 \mathrm{e}$

(59) Song, Y.; Zhou, S.; Jin, K.; Qiao, J.; Li, D.; Xu, C.; Hu, D.; Di, J.; Li, M.; Zhang, Z.; et al. Nanoscale 2018, 10, 4077. doi: $10.1039 / \mathrm{c} 7 \mathrm{nr} 08595 \mathrm{~h}$

(60) Xu, L.; Peng, Q.; Zhu, Y.; Zhao, X.; Yang, M.; Wang, S.; Xue, F.; Yuan, Y.; Lin, Z.; Xu, F.; et al. Nanoscale 2019, 11, 8124. doi: $10.1039 / \mathrm{c} 9 \mathrm{nr} 00611 \mathrm{~g}$

(61) Mu, J.; de Andrade, M. J.; Fang, S.; Wang, X.; Gao, E.; Li, N.; Kim, S. H.; Wang, H.; Hou, C.; Zhang, Q.; et al. Science 2019, 365, 150. doi: $10.1126 /$ science.aaw 2403

(62) Ren, M.; Qiao, J.; Wang, Y.; Wu, K.; Dong, L.; Shen, X.; Zhang, H.; Yang, W.; Wu, Y.; Yong, Z.; et al. Small 2021, 17, e2006181. doi: $10.1002 / \mathrm{smll} .202006181$

(63) Wang, Y.; Qiao, J.; Wu, K.; Yang, W.; Ren, M.; Dong, L.; Zhou, Y.; Wu, Y.; Wang, X.; Yong, Z.; et al. Mater. Horiz. 2020, 7, 304. doi: $10.1039 / \mathrm{d} 0 \mathrm{mh} 01352 \mathrm{~h}$

(64) Aliev, A. E.; Oh, J.; Kozlov, M. E.; Kuznetsov, A. A.; Fang, S.; Fonseca, A. F.; Ovalle, R.; Lima, M. D.; Haque, M. H.; Gartstein, Y. N.; et al. Science 2009, 323, 1575. doi: 10.1126/science.1168312

(65) Baughman, R. H.; Cui, C. X.; Zakhidov, A. A.; Iqbal, Z.; Barisci, J. N.; Spinks, G. M.; Wallace, G. G.; Mazzoldi, A.; De Rossi, D.; Rinzler, A. G.; et al. Science 1999, 284, 1340. doi: $10.1126 /$ science. 284.5418 .1340

(66) Xie, X.; Qu, L.; Zhou, C.; Li, Y.; Zhu, J.; Bai, H.; Shi, G.; Dai, L. ACS Nano 2010, 4, 6050. doi: 10.1021/nn101563x

(67) Park, S.; An, J.; Suk, J. W.; Ruoff, R. S. Small 2010, 6, 210. doi: $10.1002 / \mathrm{smll} .200901877$

(68) Lerf, A.; Buchsteiner, A.; Pieper, J.; Schöttl, S.; Dekany, I.; Szabo, T.; Boehm, H. P. J. Phys. Chem. Solids 2006, 67, 1106. doi: 10.1016/j.jpcs.2006.01.031

(69) Sun, G.; Pan, Y.; Zhan, Z.; Zheng, L.; Lu, J.; Pang, J. H. L.; Li, L.; Huang, W. J. Phys. Chem. C 2011, 115, 23741. doi: $10.1021 / \mathrm{jp} 207986 \mathrm{~m}$

(70) Mu, J.; Hou, C.; Zhu, B.; Wang, H.; Li, Y.; Zhang, Q. Sci. Rep. 2015, 5, 9503. doi: 10.1038/srep09503

(71) Han, D. D.; Zhang, Y. L.; Jiang, H. B.; Xia, H.; Feng, J.; Chen, Q. D.; Xu, H. L.; Sun, H. B. Adv. Mater. 2015, 27, 332. doi: 10.1002/adma.201403587

(72) Han, D. D.; Zhang, Y. L.; Liu, Y.; Liu, Y. Q.; Jiang, H. B.; Han, B.; Fu, X. Y.; Ding, H.; Xu, H. L.; Sun, H. B. Adv. Funct. Mater. 2015, 
25, 4548. doi: 10.1002/adfm.201501511

(73) Xu, G.; Chen, J.; Zhang, M.; Shi, G. Sens. Actuators B 2017, 242, 418. doi: 10.1016/j.snb.2016.11.068

(74) Cheng, H.; Zhao, F.; Xue, J.; Shi, G.; Jiang, L.; Qu, L. ACS Nano 2016, 10, 9529. doi: 10.1021/acsnano.6b04769

(75) Liu, J.; Wang, Z.; Xie, X.; Cheng, H.; Zhao, Y.; Qu, L. J. Mater. Chem. 2012, 22, 4015. doi: 10.1039/c2jm15266e

(76) Mukai, K.; Yamato, K.; Asaka, K.; Hata, K.; Oike, H. Sens. Actuators B 2012, 161, 1010. doi: 10.1016/j.snb.2011.11.084

(77) Shi, Q.; Hou, C.; Wang, H.; Zhang, Q.; Li, Y. Chem. Commun. 2016, 52, 5816. doi: 10.1039/c6cc01590e

(78) Xu, G.; Zhang, M.; Zhou, Q.; Chen, H.; Gao, T.; Li, C.; Shi, G. Nanoscale 2017, 9, 17465. doi: 10.1039/c7nr07116g

(79) Chen, L.; Weng, M.; Zhou, Z.; Zhou, Y.; Zhang, L.; Li, J.; Huang, Z.; Zhang, W.; Liu, C.; Fan, S. ACS Nano 2015, 9, 12189. doi: 10.1021/acsnano.5b05413

(80) Hu, Y.; Lan, T.; Wu, G.; Zhu, Z.; Chen, W. Nanoscale 2014, 6, 12703. doi: $10.1039 / \mathrm{c} 4 \mathrm{nr} 02768 \mathrm{j}$

(81) Chen, L.; Liu, C.; Liu, K.; Meng, C.; Hu, C.; Wang, J.; Fan, S. ACS Nano 2011, 5, 1588. doi: 10.1021/nn102251a

(82) Wen, Y.; Wu, M.; Zhang, M.; Li, C.; Shi, G. Adv. Mater. 2017, 29, 1702831. doi: 10.1002/adma.201702831

(83) Wan, S.; Peng, J.; Jiang, L.; Cheng, Q. Adv. Mater. 2016, 28, 7862. doi: 10.1002/adma.201601934

(84) Kim, J.; Jeon, J. H.; Kim, H. J.; Lim, H.; Oh, I. K. ACS Nano 2014, 8, 2986. doi: $10.1021 / \mathrm{nn} 500283 \mathrm{q}$

(85) Li, J.; Ma, W.; Song, L.; Niu, Z.; Cai, L.; Zeng, Q.; Zhang, X.; Dong, H.; Zhao, D.; Zhou, W.; et al. Nano Lett. 2011, 11, 4636. doi: $10.1021 / \mathrm{nl} 202132 \mathrm{~m}$

(86) Im, K. H.; Choi, H. J. Korean Phys. Soc.2014, 64, 623. doi: $10.3938 / \mathrm{jkps} .64 .623$

(87) Liu, S.; Liu, Y.; Cebeci, H.; de Villoria, R. G.; Lin, J. H.; Wardle, B. L.; Zhang, Q. M. Adv. Funct. Mater. 2010, 20, 3266. doi: 10.1002/adfm.201000570

(88) Kim, J.; Bae, S. H.; Kotal, M.; Stalbaum, T.; Kim, K. J.; Oh, I. K. Small 2017, 13, 1701314. doi: 10.1002/smll.201701314

(89) Shian, S.; Diebold, R. M.; Clarke, D. R. Opt. Express 2013, 21, 8669. doi: 10.1364/OE.21.008669

(90) Yuan, W.; Hu, L. B.; Yu, Z. B.; Lam, T.; Biggs, J.; Ha, S. M.; Xi, D. J.; Chen, B.; Senesky, M. K.; Grüner, G.; et al. Adv. Mater. 2008, 20, 621. doi: 10.1002/adma.200701018
(91) Kinloch, I. A.; Suhr, J.; Lou, J.; Young, R. J.; Ajayan, P. M. Science 2018, 362, 547. doi: 10.1126/science.aat7439

(92) Yuan, J.; Neri, W.; Zakri, C.; Merzeau, P.; Kratz, K.; Lendlein, A.; Poulin, P. Science 2019, 365, 155. doi: 10.1126/science.aaw3722

(93) Wang, Y.; Sun, L. Z. Appl. Phys. Lett. 2017, 111, 161904. doi: 10.1063/1.4997092

(94) Zhang, F.; Li, T.; Luo, Y. Compos. Sci. Technol. 2018, 156, 151. doi: 10.1016/j.compscitech.2017.12.016

(95) Kim, D.; Lee, H. S.; Yoon, J. Sci. Rep. 2016, 6, 20921. doi: $10.1038 /$ srep20921

(96) Kim, H.; Ahn, S. K.; Mackie, D. M.; Kwon, J.; Kim, S. H.; Choi, C.; Moon, Y. H.; Lee, H. B.; Ko, S. H. Mater. Today 2020, 41, 243. doi: 10.1016/j.mattod.2020.06.005

(97) Mirvakili, S. M.; Hunter, I. W. Adv. Mater. 2017, 29, 1604734. doi: 10.1002/adma.201604734

(98) Li, Q.; Liu, C.; Lin, Y. H.; Liu, L.; Jiang, K.; Fan, S. ACS Nano 2015, 9 , 409. doi: 10.1021/nn505535k

(99) Oh, J. H.; Anas, M.; Barnes, E.; Moores, L. C.; Green, M. J. Adv. Eng. Mater. 2021, 23, 2000873. doi: 10.1002/adem.202000873

(100) Ling, Y.; Pang, W.; Li, X.; Goswami, S.; Xu, Z.; Stroman, D.; Liu, Y.; Fei, Q.; Xu, Y.; Zhao, G.; et al. Adv. Mater. 2020, 32, 1908475. doi: 10.1002/adma.201908475

(101) Han, B.; Zhang, Y. L.; Zhu, L.; Li, Y.; Ma, Z. C.; Liu, Y. Q.; Zhang, X. L.; Cao, X. W.; Chen, Q. D.; Qiu, C. W.; et al. Adv. Mater. 2019, 31, 1806386. doi: 10.1002/adma.201806386

(102) Mu, J.; Hou, C.; Wang, H.; Li, Y.; Zhang, Q.; Zhu, M. Sci. Adv. 2015, 1 , e1500533. doi: 10.1126/sciadv. 1500533

(103) Dong, Y.; Wang, J.; Guo, X.; Yang, S.; Ozen, M. O.; Chen, P.; Liu, X.; Du, W.; Xiao, F.; Demirci, U.; Liu, B. F. Nat. Commun. 2019, 10, 4087. doi: 10.1038/s41467-019-12044-5

(104) Chen, L.; Weng, M.; Zhou, P.; Huang, F.; Liu, C.; Fan, S.; Zhang, W. Adv. Funct. Mater. 2019, 29, 1806057. doi: $10.1002 / \mathrm{adfm} .201806057$

(105) Zhao, H.; Hu, R.; Li, P.; Gao, A.; Sun, X.; Zhang, X.; Qi, X.; Fan, Q.; Liu, Y.; Liu, X.; et al. Nano Energy 2020, 76, 104926. doi: 10.1016/j.nanoen.2020.104926

(106) Wang, X. Q.; Chan, K. H.; Cheng, Y.; Ding, T.; Li, T.; Achavananthadith, S.; Ahmet, S.; Ho, J. S.; Ho, G. W. Adv. Mater. 2020, 32, e2000351. doi: 10.1002/adma.202000351

(107) Xiao, Y.; Lin, J.; Xiao, J.; Weng, M.; Zhang, W.; Zhou, P.; Luo, Z.; Chen, L. Nanoscale 2021, 13, 6259. doi: 10.1039/d0nr09210j 